\title{
Surface Raman scattering of the light in antiferromagnetic chromium*
}

\author{
C. B. Cuden and R. Mota \\ Instituto de Física, Universidade Federal da Bahia, Salvador, Bahia, Brasil
}

(Received 31 May 1978; revised manuscript received 10 January 1979)

\begin{abstract}
Raman scattering of light from the plane surface of antiferromagnetic chromium is discussed in terms of conventional second-order time-dependent perturbation theory. The scattering efficiency as a function of frequency and temperature is calculated from the scattering of light by one-electron, one-hole, and electron-hole pair excitations. It is shown that the unphysical divergence of the scattering cross section for the photon energy transfer equal to twice the antiferromagnetic energy gap found by Kwok, Woo, and Jha is removed by taking properly into account the statistical coherence factors.
\end{abstract}

\section{INTRODUCTION}

The electronic Raman scattering was predicted theoretically by Smekal ${ }^{1}$ already in 1923 . In 1956 Khaikin and Bykov ${ }^{2}$ tried to observe the energy gap in superconducting lead using light-scattering techniques. However, they were not able to observe the spectral distribution of the scattered light because of the small amount of scattering associated mainly with the fact that the skin effect allows the light to penetrate only into a very thin surface layer of metal. The observation can be greatly impaired also by a background scattering and strong absorption.

Only after the successful scattering experiments in heavily doped $n$-type semiconductors ${ }^{3}$ the subject received more attention in recent literature. ${ }^{4,5}$

Experiments of Raman scattering from the surface of metals were first reported in 1968 by Feldman, Parker, and Ashkin ${ }^{6}$ and the appropriate theory has been presented by Mills et al. ${ }^{7}$

With the improved experimental technique, Moora$\operatorname{dian}^{8}$ observed that many metals exhibit a weak, broadband electronic luminescence when exposed to intense laser monochromatic light.

In 1970 Raman-scattering experiments on tungsten bronzes have been reported by Scott et al. ${ }^{9}$ As pointed out by Tilley, ${ }^{10}$ these authors studied scattering at frequency shifts above $200 \mathrm{~cm}^{-1}$ so that they probed the optical-phonon spectrum.

The frequency shifts of interest in superconductivity are of the order of the energy gap $\Delta$, that is, a few wave numbers. In fact the experiment by Fraas et al. ${ }^{11}$ has shown that in superconducting $\mathrm{Nb}_{3} \mathrm{Sn}$, scattering disappeared in the frequency region $\omega_{\text {laser }}>\omega>\omega_{\text {laser }}-2 \Delta$.

Furthermore, their polarization measurements at $150 \mathrm{~cm}^{-1}$ indicated little intensity change with polarization indicating that the intermediate interband transitions are important.

In the theoretical work on Raman scattering of light off a superconductor by Abrikosov and Falkovskii, ${ }^{12}$ Maradudin and Tong, ${ }^{13}$ Tilley, ${ }^{14}$ Dos Reis and Luzzi, ${ }^{15}$ only intraband electronic intermediate transitions in a superconductor were considered. Their work exhibits a gap in the spectrum, but disagree completely with the temperature and frequency dependence of the reflected light off a superconducting $\mathrm{Nb}_{3} \mathrm{Sn}$, found in the experiment by Fraas et al.

In Ref. 16 Raman scattering of light in pure superconductors was discussed. It was demonstrated by means of standard formulation of Raman scattering of light that the main contribution to the intensity of the inelastically scattered light in a superconductor comes from the electronic interband intermediate transitions whose energy difference is close to the energy of the incident light and not from the intermediate intraband transitions as presented in earlier literature. For the specific case of $\mathrm{Nb}_{3} \mathrm{Sn}$, good agreement between the theory and experimental work by Fraas, Porto, and Williams was found.

In Ref. 17, the scattering efficiency was formulated in terms of Kubo's nonlinear-response theory in a form suitable for systematic diagrammatic expansion. It was shown that the main contribution to the intensity of the inelastically scattered light comes from the unscreened fluctuations in transverse current, and not from density fluctuations as considered in earlier work. Frequency and temperature dependence of the scattering efficiency was obtained for the case of tin.

In Ref. 18 Raman scattering of light off a superconducting alloy containing low concentration of paramagnetic impurities was discussed. For the specific case of $\mathrm{Fe}$ impurities in In films, the scattering efficiency as a function of frequency and temperature was calculated for various impurity concentrations.

The existence of spin-orbit coupling in some semiconductors and insulators renders possible the inelastic scattering of light by spin-density fluctua- 
tions ${ }^{19,20}$ (SDF), which correspond essentially to the spin-flip electron-hole pair excitations.

In the presence of electronic coherent states in superconductors and in metals with itinerant antiferromagnetic ordering, the scattering of light by excitations of bound Cooper pairs and bound electron-hole pairs in triplet state, respectively, is possible, even in the absence of electron spin-orbit coupling.

In Ref. 21 the inelastic scattering of light in antiferromagnetic chromium was discussed. At zero temperature the scattering cross section was found divergent for the photon energy transfer equal to twice the antiferromagnetic energy gap. This is clearly a nonphysical result, and in this paper we propose to reinvestigate the Raman scattering of light in itinerant antiefrromagnetic chromium.

In Sec. II we give a BCS-like effective Hamiltonian, describing the static spin-density-wave state, based on Lomer's ${ }^{22}$ two-band model for $\mathrm{Cr}$.

In Sec. III we present a general formalism for the inelastic scattering of light by electronic excitations from the plane surface of a metal. In Sec. IV we calculate the scattering efficiency for the scattering of light by single-electron and -hole excitations which are thermally excited out of the "condensate" and exist at finite temperature only. In Sec. $\mathrm{V}$ we calculate the scattering efficiency for the scattering of light by excitations of bound electron-hole pairs in triplet state. In Sec. VI we give the total scattering efficiency. And finally, in Sec. VII, we compare our results with those obtained in Ref. 21 and give our concluding remarks.

\section{SYSTEM HAMILTONIAN}

In pure chromium, below the Néel temperature $T_{N}=312^{\circ} \mathrm{K}$, neutron scattering experiments ${ }^{23}$ have shown the existence of local magnetic moments. The scattering cross section for the inelastically scattered neutrons by spin-flip excitations exhibits a monotonically decreasing temperature-dependent energy gap, with its maximum value at $T=0^{\circ} \mathrm{K}$ given approximately by $2 g \simeq 3.5 k_{B} T$.

Band calculations ${ }^{24,25}$ have shown that there is a large roughly octahedral pocket of electrons at the center of the zone and a pocket of holes centered at the point $H$ at the edge of the zone. The shape and the size of the two bands were found very similar.

At temperatures below the critical Néel temperature the nearly degenerate conduction electrons and holes with opposite spins form bound pairs, and as a result an itinerant antiferromagnetic phase appears.

Following Lomer ${ }^{22}$ and Overhouser, ${ }^{26}$ let us assume that the antiferromagnetic transition is the result of attractive interaction between nearly degenerate, opposite-spin electron and hole states from two bands separated by a wave vector of antiferromagnetic ordering $\vec{Q}$. In this case, below the Néel temperature, the system may be well represented by the approximate Hamiltonian of the form

$$
\begin{aligned}
H= & \sum_{\overrightarrow{\mathrm{p}} \sigma} \epsilon_{1}(\overrightarrow{\mathrm{p}}) c_{1 \overrightarrow{\mathrm{p}} \sigma}^{\dagger} c_{1 \overrightarrow{\mathrm{p}} \sigma}+\sum_{\overrightarrow{\mathrm{p}} \sigma} \epsilon_{2}(\overrightarrow{\mathrm{p}}) c_{2 \overrightarrow{\mathrm{p}} \sigma}^{\dagger} c_{2 \overrightarrow{\mathrm{p}} \sigma} \\
& +\sum_{\overrightarrow{\mathrm{p}}} g(\overrightarrow{\mathrm{p}})\left(c_{1 \overrightarrow{\mathrm{p}} \mid}^{\dagger} c_{2 \overrightarrow{\mathrm{p}}+\overrightarrow{\mathrm{Q}} \mid}+c_{2 \overrightarrow{\mathrm{p}}+\overrightarrow{\mathrm{Q}} \mid}^{\dagger} c_{1 \overrightarrow{\mathrm{p}} \mid}+c_{2-\overrightarrow{\mathrm{p}}-\overrightarrow{\mathrm{Q}} \mid}^{\dagger} c_{1-\overrightarrow{\mathrm{p}} !}+c_{1-\overrightarrow{\mathrm{p}} \mid}^{\dagger} c_{2-\overrightarrow{\mathrm{p}}-\overrightarrow{\mathrm{Q}} \mid}\right)+\sum_{n \geqslant 3 \overrightarrow{\mathrm{p}} \sigma} \epsilon_{n}(\overrightarrow{\mathrm{p}}) c_{n \overrightarrow{\mathrm{p}} \sigma}^{\dagger} c_{n \overrightarrow{\mathrm{p}} \sigma},
\end{aligned}
$$

where $\epsilon_{1}(\vec{p})$ and $\epsilon_{2}(\vec{p})$ are the kinetic energies of conduction electrons in bands 1 and 2 , respectively, and have the property that $\epsilon_{1}(\vec{p}) \simeq \epsilon_{2}(\vec{p}+\vec{Q})$ for an appreciable region in $\vec{p}$ space near the Fermi surface in band 1. By inversion symmetry, one has $\epsilon_{1}(-\vec{p})=\epsilon_{2}(-\vec{p}-\vec{Q}) . \quad \epsilon_{n}(\vec{p})$ for $n \geqslant 3$ is the energy of an electron in band $n$.

In Eq. (2.1) $g(\vec{p})$ represents the amplitude of the self-consistent spin-density wave (SDW),

$$
\begin{aligned}
g(\overrightarrow{\mathrm{p}})=-\sum_{\overrightarrow{\mathrm{p}}^{\prime}}[ & V\left(\overrightarrow{\mathrm{p}}^{\prime}-\overrightarrow{\mathrm{p}}\right)\left\langle 1 \overrightarrow{\mathrm{p}}^{\prime} \mid 1 \overrightarrow{\mathrm{p}}\right\rangle\left\langle 2 \overrightarrow{\mathrm{p}}+\overrightarrow{\mathrm{Q}} \mid 2 \overrightarrow{\mathrm{p}}^{\prime}+\overrightarrow{\mathrm{Q}}\right\rangle \\
& +V\left(\overrightarrow{\mathrm{p}}-\overrightarrow{\mathrm{p}}^{\prime}\right)\left\langle 2 \overrightarrow{\mathrm{p}}+\overrightarrow{\mathrm{Q}} \mid 1 \overrightarrow{\mathrm{p}}^{\prime}+\overrightarrow{\mathrm{Q}}\right\rangle \\
& \left.\times\left\langle 2 \overrightarrow{\mathrm{p}}^{\prime} \mid 1 \overrightarrow{\mathrm{p}}\right\rangle\right]\left\langle c_{1 \overrightarrow{\mathrm{p}}^{\prime} \dagger}^{\dagger} c_{2 \overrightarrow{\mathrm{p}}^{\prime}+\overrightarrow{\mathrm{Q}} \downarrow}\right\rangle
\end{aligned}
$$

where

$$
\left\langle n \overrightarrow{\mathrm{p}} \mid n^{\prime} \overrightarrow{\mathrm{p}}^{\prime}\right\rangle=\int u_{n \overrightarrow{\mathrm{p}}}^{*}(\overrightarrow{\mathrm{r}}) u_{n^{\prime} \overrightarrow{\mathrm{p}}^{\prime}}(\overrightarrow{\mathrm{r}}) d^{3} r .
$$

$V(\vec{q})$ is the Fourier transform of the screened Coulomb interaction and $u_{n \vec{p}}(\vec{r})$ is the periodic part of the Bloch wave function. $\langle\cdots\rangle$ represents the usual thermal average,

$$
\langle\cdots\rangle=\frac{\operatorname{Tr} e^{-\beta H} \cdots}{\operatorname{Tr} e^{-\beta H}}, \beta \equiv \frac{1}{k_{B} T} .
$$

By assuming a constant effective amplitude of the self-consistent SDW one can diagonalize the Hamiltonian given by Eq. (2.1).

By using the Bogolubov transformation

$$
\begin{aligned}
& c_{1 \overrightarrow{\mathrm{p}} \mid}=u_{\overrightarrow{\mathrm{p}}} \alpha_{\overrightarrow{\mathrm{p}}}+v_{\overrightarrow{\mathrm{p}}} \beta_{\frac{\mathrm{p}}{\dagger}}^{\dagger}, \\
& c_{2 \overrightarrow{\mathrm{p}}+\overrightarrow{\mathrm{Q}} !}=-v_{\overrightarrow{\mathrm{p}}} \alpha_{\overrightarrow{\mathrm{p}}}+u_{\overrightarrow{\mathrm{p}}} \beta \frac{\overrightarrow{\mathrm{p}}}{\dagger}, \\
& c_{1-\overrightarrow{\mathrm{p}} \mid}=-\bar{v}_{-\overrightarrow{\mathrm{p}}-\overrightarrow{\mathrm{Q}}} \bar{\alpha}_{-\overrightarrow{\mathrm{p}}-\overrightarrow{\mathrm{Q}}}+\bar{u}_{-\overrightarrow{\mathrm{p}}-\overrightarrow{\mathrm{Q}}} \bar{\beta}_{-\overrightarrow{\mathrm{p}}-\overrightarrow{\mathrm{Q}}}^{\dagger}, \\
& c_{2-\overrightarrow{\mathrm{p}}-\overrightarrow{\mathrm{Q}} \mid}=\bar{u}_{-\overrightarrow{\mathrm{p}}-\overrightarrow{\mathrm{Q}}} \bar{\alpha}_{-\overrightarrow{\mathrm{p}}-\overrightarrow{\mathrm{Q}}}+\bar{v}_{-\overrightarrow{\mathrm{p}}-\overrightarrow{\mathrm{Q}}} \bar{\beta}_{-\overrightarrow{\mathrm{p}}-\overrightarrow{\mathrm{Q}}}^{\dagger},
\end{aligned}
$$


the Hamiltonian (2.1) can be cast into the simple form

$$
\begin{aligned}
H= & \sum_{\overrightarrow{\mathrm{p}}} E_{\alpha}(\overrightarrow{\mathrm{p}}) \alpha_{\overrightarrow{\mathrm{p}}}^{\dagger} \alpha_{\overrightarrow{\mathrm{p}}}+\sum_{\overrightarrow{\mathrm{p}}} E_{\beta}(\overrightarrow{\mathrm{p}}) \beta_{\overrightarrow{\mathrm{p}}}^{\dagger} \beta_{\overrightarrow{\mathrm{p}}} \\
& +\sum_{\overrightarrow{\mathrm{p}}} E_{\overline{\boldsymbol{\alpha}}}(-\overrightarrow{\mathrm{p}}-\overrightarrow{\mathrm{Q}}) \bar{\alpha}_{-\overrightarrow{\mathrm{p}}-\overrightarrow{\mathrm{Q}}}^{\dagger} \bar{\alpha}_{-\overrightarrow{\mathrm{p}}-\overrightarrow{\mathrm{Q}}} \\
& +\sum_{\overrightarrow{\mathrm{p}}} E_{\bar{\beta}}(-\overrightarrow{\mathrm{p}}-\overrightarrow{\mathrm{Q}}) \bar{\beta}_{-\overrightarrow{\mathrm{p}}-\overrightarrow{\mathrm{Q}}}^{\dagger} \bar{\beta}_{-\overrightarrow{\mathrm{p}}-\overrightarrow{\mathrm{Q}}} \\
& +\sum_{n \geqslant 3 \overrightarrow{\mathrm{p}} \sigma} \epsilon_{n}(\overrightarrow{\mathrm{p}}) c_{n \overrightarrow{\mathrm{p}} \sigma}^{\dagger} c_{n \overrightarrow{\mathrm{p}} \sigma},
\end{aligned}
$$

with

$$
\begin{aligned}
E_{\alpha}(\overrightarrow{\mathrm{p}}) & =\frac{1}{2}\left[\epsilon_{1}(\overrightarrow{\mathrm{p}})+\epsilon_{2}(\overrightarrow{\mathrm{p}}+\overrightarrow{\mathrm{Q}})+\Omega(\overrightarrow{\mathrm{p}})\right] \\
& =E_{\bar{\alpha}}(-\overrightarrow{\mathrm{p}}-\overrightarrow{\mathrm{Q}}) \\
E_{\beta}(\overrightarrow{\mathrm{p}}) & =\frac{1}{2}\left[\epsilon_{1}(\overrightarrow{\mathrm{p}})+\epsilon_{2}(\overrightarrow{\mathrm{p}}+\overrightarrow{\mathrm{Q}})-\Omega(\overrightarrow{\mathrm{p}})\right] \\
& =E_{\bar{\beta}}(-\overrightarrow{\mathrm{p}}-\overrightarrow{\mathrm{Q}}), \\
\Omega(\overrightarrow{\mathrm{p}}) & =\left\{\left[\epsilon_{1}(\overrightarrow{\mathrm{p}})-\epsilon_{2}(\overrightarrow{\mathrm{p}})\right]^{2}+4 g^{2}\right\}^{1 / 2},
\end{aligned}
$$

where $E_{\alpha}(\overrightarrow{\mathrm{p}})$ and $E_{\beta}(\overrightarrow{\mathrm{p}})$ are the energies of the elementary excitations in an itinerant antiferromagnet, and $g$ is the temperature-dependent order parameter determined by the following BCS-like equation:

$$
1=\bar{V} \sum_{\overrightarrow{\mathrm{p}}} \frac{f\left(E_{\alpha}(\overrightarrow{\mathrm{p}})\right)-f\left(E_{\beta}(\overrightarrow{\mathrm{p}})\right)}{E_{\beta}(\overrightarrow{\mathrm{p}})-E_{\alpha}(\overrightarrow{\mathrm{p}})}
$$

$\bar{V}$ denotes a constant effective Coulomb interaction and is given by some appropriate average value of

$$
\begin{aligned}
& {\left[V\left(\overrightarrow{\mathrm{p}}^{\prime}-\overrightarrow{\mathrm{p}}\right)\left\langle 1 \overrightarrow{\mathrm{p}}^{\prime} \mid 1 \overrightarrow{\mathrm{p}}\right\rangle\left\langle 2 \overrightarrow{\mathrm{p}}+\overrightarrow{\mathrm{Q}} \mid 2 \overrightarrow{\mathrm{p}}^{\prime}+\overrightarrow{\mathrm{Q}}\right\rangle\right.} \\
& \left.\quad+V\left(\overrightarrow{\mathrm{p}}-\overrightarrow{\mathrm{p}}^{\prime}\right)\left\langle 2 \overrightarrow{\mathrm{p}}+\overrightarrow{\mathrm{Q}} \mid 1 \overrightarrow{\mathrm{p}}^{\prime}+\overrightarrow{\mathrm{Q}}\right\rangle\left\langle 2 \overrightarrow{\mathrm{p}}^{\prime} \mid 1 \overrightarrow{\mathrm{p}}\right\rangle\right] \\
& u_{\overrightarrow{\mathrm{p}}}, v_{\overrightarrow{\mathrm{p}}}, \bar{u}_{-\overrightarrow{\mathrm{p}}-\overrightarrow{\mathrm{Q}}}, \text { and } \bar{v}_{-\overrightarrow{\mathrm{p}}-\overrightarrow{\mathrm{Q}}} \text { are given by } \\
& \quad u_{\overrightarrow{\mathrm{p}}}^{2}=\frac{1}{2}\left\{1+\left[\epsilon_{1}(\overrightarrow{\mathrm{p}})-\epsilon_{2}(\overrightarrow{\mathrm{p}}+\overrightarrow{\mathrm{Q}})\right] / \Omega(\overrightarrow{\mathrm{p}})\right\} \\
& \quad v_{\overrightarrow{\mathrm{p}}}^{2}=\frac{1}{2}\left\{1-\left[\epsilon_{1}(\overrightarrow{\mathrm{p}})-\epsilon_{2}(\overrightarrow{\mathrm{p}}+\overrightarrow{\mathrm{Q}})\right] / \Omega(\overrightarrow{\mathrm{p}})\right\} \\
& \bar{u}_{-\overrightarrow{\mathrm{p}}-\overrightarrow{\mathrm{Q}}}=v_{\overrightarrow{\mathrm{p}}} \\
& \bar{v}_{-\overrightarrow{\mathrm{p}}-\overrightarrow{\mathrm{Q}}}=u_{\overrightarrow{\mathrm{p}}}
\end{aligned}
$$

$\alpha$ 's, $\beta$ 's, $\bar{\alpha}$ 's, $\bar{\beta}$ 's, and c's satisfy the usual Fermi anticommutation relations. The critical Néel temperature can be determined by setting $g=0$ in the Eq.

(2.10). It is given by

$$
T_{N}=T_{B} e^{-1 / \lambda},
$$

where $\lambda=N(0) \bar{V}, N(0)$ is the density of states at the Fermi energy, and $k_{B} T_{B}$ is of the order of band energy.

Mathematically the presented model is equivalent to the BCS model of superconductivity. Physically, however, the two models are very different. The condensate is now composed of bound electron-hole pairs in triplet state rather than Cooper electronelectron pairs.

As there always exist a nonmagnetic part of the Fermi surface, there are of course none of the superfluid properties associated with superconductivity.

Based on the Lomer and Overhauser theory and using a simplified model for the band structure, Fedders and Martin ${ }^{27}$ have calculated specific heat, longitudinal collective mode, spin susceptibility, and electromagnetic absorption in $\mathrm{Cr}$.

\section{SCATTERING EFFICIENCY}

Scattering of light off a metallic surface was considered by Mills et $a l^{7}$ The interaction of light with the electrons responsible for the Raman scattering takes place within the penetration region of the light in the metal.

The fact that the fields outside, which are the ones measured, differ from those inside the metal can be taken into account by solving the usual boundaryvalue problem at the metal surface and introducing Fresnel correction factors.

We assume the light is reflected from the plane surface of a metal filling the semi-infinite volume $z<0$. See Fig. 1 .

The dielectric constant $\epsilon(\omega)$ of the sample is assumed local and isotropic.

Following the results of Ref. 17 and 28 the scattering efficiency defined as the ratio between the number of scattered photons in the frequency interval $(\omega, \omega+d \omega)$, solid angle $d \Omega$, and volume $V_{D}$ and the number of incident photons of frequency $\omega_{0}$ fal-

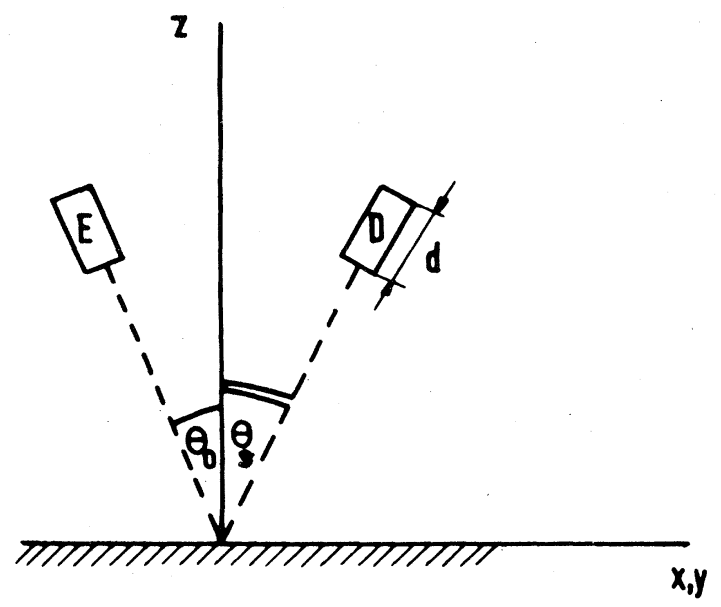

FIG. 1. Light generated in the emitter region $V_{E}$ is scattered in the sample, which occupies the half-space $z<0$, and reflected light is detected in the detector region $V_{D}$ far from the sample. 
ling on the surface $S$ in time $t$ can be written in the form ${ }^{17,28}$

$$
\frac{1}{\phi_{0}} \frac{d^{2} \phi}{d \omega d \Omega}=\frac{4 \omega_{s} \cos ^{2} \theta_{s}}{S \omega_{0} c^{4} \cos \theta_{0}} \sum_{\substack{\mu \nu \eta^{\xi} \xi \\ \mu^{\prime} \nu^{\prime} \eta^{\prime} \xi^{\prime}}} e_{s \mu} e_{s \nu} D_{\mu \mu^{\prime}}\left(\overrightarrow{\mathrm{k}}_{\|}, \omega_{s}\right) D_{\nu \nu^{\prime}}^{*}\left(\overrightarrow{\mathrm{k}}_{\|,}, \omega_{s}\right) G_{\mu^{\prime} \nu^{\prime} \eta^{\prime} \xi^{\prime}}\left(\overrightarrow{\mathrm{k}}, \overrightarrow{\mathrm{k}}_{0}\right) T_{\eta^{\prime} \eta} T_{\xi^{\prime} \xi}^{*} e_{0 \eta} e_{0 \xi},
$$

where

$$
G_{\mu \nu \eta \xi}\left(\overrightarrow{\mathrm{k}}, \overrightarrow{\mathrm{k}}_{0}\right)=\sum_{i f}\left\langle f\left|t_{\mu \eta}\left(\overrightarrow{\mathrm{k}}, \overrightarrow{\mathrm{k}}_{0}\right)\right| i\right\rangle\left\langle f\left|t_{\nu \xi}\left(\overrightarrow{\mathrm{k}}, \overrightarrow{\mathrm{k}}_{0}\right)\right| i\right\rangle^{*}\left(P_{i}-P_{f}\right) \delta\left(\omega-\left|E_{f}-E_{i}\right|\right),
$$

and

$$
\begin{aligned}
\langle f| t_{\mu \nu}\left(\overrightarrow{\mathrm{k}}, \overrightarrow{\mathrm{k}}_{0}|i\rangle=S^{-1 / 2} \int_{z_{1}, z_{2}<0}\right. & d \overrightarrow{\mathrm{r}}_{1} d \overrightarrow{\mathrm{r}}_{2} e^{i\left(\left(\overrightarrow{\mathrm{k}}_{0 \mid l_{1}}-\overrightarrow{\mathrm{k}}_{1 \mid \mathrm{r}_{2}}\right)+\gamma_{0} z_{1}+\gamma_{s} z_{2}\right]} \\
\times & \left\{\delta_{\mu \nu} \frac{e^{2}}{m}\left\langle f\left|n\left(\overrightarrow{\mathrm{r}}_{1}\right)\right| i\right\rangle \delta\left(\overrightarrow{\mathrm{r}}_{1}-\overrightarrow{\mathrm{r}}_{2}\right)\right. \\
& \left.+\sum_{l}\left[\frac{\left\langle f\left|j_{\mu}\left(\overrightarrow{\mathrm{r}}_{2}\right)\right| l\right\rangle\left\langle l\left|j_{\nu}\left(\overrightarrow{\mathrm{r}}_{1}\right)\right| i\right\rangle}{E_{i}-E_{l}+\omega_{0}}+\frac{\left\langle f\left|j_{\nu}\left(\overrightarrow{\mathrm{r}}_{1}\right)\right| l\right\rangle\left\langle l\left|j_{\mu}\left(\overrightarrow{\mathrm{r}}_{2}\right)\right| i\right\rangle}{E_{i}-E_{l}-\omega_{s}}\right]\right\} .
\end{aligned}
$$

From Eq. (3.3) we see that within the framework of an independent-quasiparticle model, only the second term in the curly bracket contributes to the inelastic scattering of light.

The frequency of the incident light $\omega_{0}$, scattered light $\omega_{s}$, and the transferred frequency $\omega$ are related to the corresponding wave vectors $\overrightarrow{\mathrm{k}_{0}}, \overrightarrow{\mathrm{k}}$, and $\overrightarrow{\mathrm{q}}$ in the usual way,

$$
\begin{aligned}
& \omega_{0}=c\left|\overrightarrow{\mathrm{k}}_{0}\right|=c k_{0}, \quad \omega_{s}=c|\overrightarrow{\mathbf{k}}|=c k, \\
& \omega=c|\overrightarrow{\mathrm{q}}|=c q, \quad \overrightarrow{\mathbf{q}}=\overrightarrow{\mathrm{k}}_{0}-\overrightarrow{\mathrm{k}} .
\end{aligned}
$$

The tensor $\underline{T}$ and the function $D\left(\overrightarrow{\mathrm{k}}_{\|}, c k\right)$ in dyadic form are given by ${ }^{17}$

$$
\begin{aligned}
& T_{\alpha \alpha^{\prime}}=T_{\alpha} \delta_{\alpha \alpha^{\prime}}, \quad T_{\|}=2\left(\epsilon-\sin ^{2} \theta_{0}\right)^{1 / 2}\left[\left(\epsilon-\sin ^{2} \theta_{0}\right)^{1 / 2}+\epsilon \cos \theta_{0}\right], \\
& T_{\perp}=2 \cos \theta_{0} /\left[\left(\epsilon-\sin ^{2} \theta_{0}\right)^{1 / 2}+\cos \theta_{0}\right], \quad T_{z}=2 \cos \theta_{0} /\left[\left(\epsilon-\sin ^{2} \theta_{0}\right)^{1 / 2}+\epsilon \cos \theta_{0}\right], \\
& \begin{array}{c}
D\left(\overrightarrow{\mathrm{k}}_{\|}, c k \operatorname{sgn} k_{\mathrm{z}}\right)=k \operatorname{sgn} k_{\mathrm{z}} \\
{\left[\gamma_{s} k_{\mathrm{z}}\left(i \gamma_{s} k^{2}-\gamma^{2} k_{z}\right)^{-1} \overrightarrow{\mathrm{u}} \overrightarrow{\mathrm{u}}+\left(i k_{\mathrm{z}}-\gamma_{s}\right)^{-1} \overrightarrow{\mathrm{v}} \overrightarrow{\mathrm{v}}-i \overrightarrow{\mathrm{k}}_{\|}^{2}\left(i \gamma_{s} k^{2}-\gamma^{2} k_{\mathrm{z}}\right)^{-1} \overrightarrow{\mathrm{z}} \overrightarrow{\mathrm{z}}\right.} \\
\left.+i k_{\mathrm{z}} k_{\|}\left(i \gamma_{s} k^{2}-\gamma^{2} k_{\mathrm{z}}\right)^{-1} \overrightarrow{\mathrm{u}} \overrightarrow{\mathrm{z}}-\gamma_{s} k_{\|}\left(i \gamma_{s} k^{2}-\gamma^{2} k_{z}\right)^{-1} \overrightarrow{\mathrm{z}} \overrightarrow{\mathrm{u}}\right],
\end{array}
\end{aligned}
$$

where $T_{\|}, T_{\perp}$ are the components of $T_{\alpha}$ parallel and perpendicular to $\overrightarrow{\mathrm{k}}_{011}$, respectively, and $\overrightarrow{\mathrm{u}}, \overrightarrow{\mathrm{v}}$, and $\overrightarrow{\mathrm{z}}$ are the unit vectors in the Cartesian coordinate system in which $\overrightarrow{\mathrm{u}}$ is taken parallel to $\overrightarrow{\mathrm{k}}_{\|}$and $\overrightarrow{\mathrm{z}}$ to the $z$ axis; the skin depths $\gamma_{0}$ and $\gamma_{s}$ of the incident and scattered light are given by

$$
\begin{aligned}
& \gamma_{0}=\left[-\epsilon\left(\omega_{0}\right) k_{0}^{2}+k_{011}^{2}\right]^{1 / 2}, \operatorname{Re} \gamma_{0}>0 \\
& \gamma_{s}=\left[-\epsilon\left(c k \operatorname{sgn} k_{z}\right) k^{2}+k^{2}\right]^{1 / 2}, \operatorname{Re} \gamma_{s}>0
\end{aligned}
$$

and

$$
\begin{aligned}
& \gamma^{2}=-\epsilon\left(c k \operatorname{sgn} k_{z}\right), \\
& \cos \theta_{0}=-k_{0 z} / k_{0}, \\
& \cos \theta_{s}=k_{z} / k, \\
& \overrightarrow{\mathrm{k}}_{\|}=\left(k_{x}, k_{y}, 0\right), \quad \overrightarrow{\mathrm{k}}_{0 \|}=\left(k_{0 x}, k_{0 y}, 0\right) .
\end{aligned}
$$

For most metals in the frequency region of interest, i.e., for $\omega<<\omega_{0}, \omega_{s}$ the dielectric constant $\epsilon=10$. In the limit $\epsilon \gg 1, D\left(\overrightarrow{\mathbf{k}}_{\|}, c k\right)$ takes the sim- ple form

$$
D\left(\overrightarrow{\mathrm{k}}_{\|}, c k\right)=\frac{k}{\gamma_{s}}\left(-(\underline{1}-\overrightarrow{\mathrm{z}} \overrightarrow{\mathrm{z}})+\frac{k_{\|}}{k_{\mathrm{z}}} \overrightarrow{\mathrm{z}} \overrightarrow{\mathrm{u}}\right) .
$$

$|i\rangle,|f\rangle,|l\rangle$ and $E_{i}, E_{f}, E_{l}$ represent initial, final, and intermediate electronic-state vectors and energies of the system. $P_{i}$ and $P_{f}$ are the thermal weight factors indicating the probability that at temperature $T$ the initial and final states are occupied, respectively.

The summation in Eq. (3.2) is over the initial and final states, and in Eq. (3.3) over the intermediate states only.

The $\mu$ vector component of the electron current density operator $j_{\mu}(\vec{r})$ and the electron number density operator $n(\vec{r})$ can be written in second quantization formalism as

$$
\begin{aligned}
& j_{\mu}(\overrightarrow{\mathrm{r}})=\sum_{n n^{\overrightarrow{\mathrm{k}} \overrightarrow{\mathrm{q}} \sigma}}\left\langle n^{\prime} \overrightarrow{\mathrm{k}}+\overrightarrow{\mathrm{q}} \sigma\left|j_{\mu}(\overrightarrow{\mathrm{r}})\right| n \overrightarrow{\mathrm{k}} \sigma\right\rangle c_{n^{\prime} \overrightarrow{\mathrm{k}}+\overrightarrow{\mathrm{q}} \sigma}^{\dagger} c_{n \overrightarrow{\mathrm{k}} \sigma}, \\
& n(\overrightarrow{\mathrm{r}})=\sum_{n n^{\prime} \overrightarrow{\mathrm{k}} \overrightarrow{\mathrm{q}} \sigma}\left\langle n^{\prime} \overrightarrow{\mathrm{k}}+\overrightarrow{\mathrm{q}} \sigma|n(\overrightarrow{\mathrm{r}})| n \overrightarrow{\mathrm{k}} \sigma\right\rangle c_{n}^{\dagger} \overrightarrow{\mathrm{k}}+\overrightarrow{\mathrm{q}} \sigma c_{n \overrightarrow{\mathrm{k}} \sigma} .
\end{aligned}
$$




\section{SCATTERING OF LIGHT BY ONE-PARTICLE EXCITATIONS}

We are primarily interested in the photon energy transfers in the range $0<\omega \leq 6 g$, i.e., in the region of low-lying electronic excitations where

$\omega=\omega_{0}-\omega_{s}<<\omega_{0}, \omega_{s}$.

In the band structure of $\mathrm{Cr}$ one can distinguish the nonmagnetic and the magnetic part of the Fermi surface. Below the Néel temperature the electrons and holes in the vicinity of the nonmagnetic part of the Fermi surface retain their normal-metal character.

The "normal-metal" scattering efficiency involves scattering of light by the electrons near the nonmagnetic part of the Fermi surface of area $A_{n}$.
On the other hand, the electrons in the vicinity of the magnetic part of the Fermi surface in band 1 of area $A_{m}$ form bound-electron-hole pairs in triplet state.

At finite temperature, some of the pairs are broken under the influence of thermal fluctuations forming the "normal fluid" component of the "condensate".

The normal fluid scattering efficiency thus involves scattering of light by quasiparticles already present because of finite temperature. The excitation energy in this case can be arbitrarily small, as in the normal metal.

One can distinguish the following four possible pairs of the initial and final states, corresponding initial- and final-state excitation energies, and thermal-weight rate factors:

$$
\begin{aligned}
& (|i\rangle,|f\rangle)=\left(\alpha_{\overrightarrow{\mathrm{p}}}^{\dagger}\left|0_{\alpha n}\right\rangle, \alpha_{\overrightarrow{\mathrm{p}}+\overrightarrow{\mathrm{q}}}^{\dagger}\left|0_{\alpha n}\right\rangle\right),\left(\beta_{\overrightarrow{\mathrm{p}}}^{\dagger}\left|0_{\beta n}\right\rangle, \beta_{\overrightarrow{\mathrm{p}}+\vec{q}}^{\dagger}\left|0_{\beta n}\right\rangle\right), \\
& \left(\bar{\alpha}_{-\overrightarrow{-}-\vec{Q}}^{\dagger}\left|0_{\bar{\alpha} n}\right\rangle, \bar{\alpha}_{-\vec{p}-\vec{Q}-\vec{q}}^{\dagger}\left|0_{\bar{\alpha} n}\right\rangle\right),\left(\bar{\beta}_{-\vec{p}-\vec{Q}}^{\dagger}\left|0_{\bar{\beta} n}\right\rangle, \bar{\beta}_{-\vec{p}-\vec{Q}-\bar{q}}^{\dagger}\left|0_{\bar{\beta} n}\right\rangle\right), \\
& \left(E_{i}, E_{f}\right)=\left(E_{\alpha}(\overrightarrow{\mathrm{p}}), E_{\alpha}(\overrightarrow{\mathrm{p}}+\overrightarrow{\mathrm{q}})\right),\left(-E_{\beta}(\overrightarrow{\mathrm{p}}),-E_{\beta}(\overrightarrow{\mathrm{p}}+\overrightarrow{\mathrm{q}})\right), \\
& \left(E_{\alpha}(\overrightarrow{\mathrm{p}}), E_{\alpha}(\overrightarrow{\mathrm{p}}+\overrightarrow{\mathrm{q}})\right),\left(-E_{\beta}(\overrightarrow{\mathrm{p}}),-E_{\beta}(\overrightarrow{\mathrm{p}}+\overrightarrow{\mathrm{q}})\right), \\
& \left(P_{i}-P_{f}\right)=\left[f\left(E_{\alpha}(\overrightarrow{\mathrm{p}})\right)-f\left(E_{\alpha}(\overrightarrow{\mathrm{p}}+\overrightarrow{\mathrm{q}})\right)\right],\left[f\left(E_{\beta}(\overrightarrow{\mathrm{p}}+\overrightarrow{\mathrm{q}})\right)-f\left(E_{\beta}(\overrightarrow{\mathrm{p}})\right)\right], \\
& {\left[f\left(E_{\alpha}(\overrightarrow{\mathrm{p}})\right)-f\left(E_{\alpha}(\overrightarrow{\mathrm{p}}+\overrightarrow{\mathrm{q}})\right)\right],\left[f\left(E_{\beta}(\overrightarrow{\mathrm{p}}+\overrightarrow{\mathrm{q}})\right)-f\left(E_{\beta}(\overrightarrow{\mathrm{p}})\right)\right],} \\
& \left|0_{\alpha n}\right\rangle \equiv\left|0_{\alpha}\right\rangle\left|0_{n}\right\rangle, \quad\left|0_{\beta n}\right\rangle \equiv\left|0_{\beta}\right\rangle\left|0_{n}\right\rangle, \quad\left|0_{\bar{\alpha} n}\right\rangle \equiv\left|0_{\bar{\alpha}}\right\rangle\left|0_{n}\right\rangle, \quad\left|0_{\bar{\beta} n}\right\rangle \equiv\left|0_{\bar{\beta}}\right\rangle\left|0_{n}\right\rangle \text {, }
\end{aligned}
$$

where the use of Eqs. (2.7) and (2.8) has been made, and $f(E)$ is the Fermi-Dirac distribution function

$$
f(E)=\frac{1}{e^{\beta E}+1} .
$$

The vacuum states $\left|0_{\alpha}\right\rangle,\left|0_{\beta}\right\rangle,\left|0_{\bar{\alpha}}\right\rangle,\left|0_{\bar{\beta}}\right\rangle$, and $\left|0_{n}\right\rangle$ are defined by

$$
\alpha_{\overrightarrow{\mathrm{p}}}\left|0_{\alpha}\right\rangle=0, \quad \beta_{\overrightarrow{\mathrm{p}}}\left|0_{\beta}\right\rangle=0, \quad \bar{\alpha}_{\overrightarrow{\mathrm{p}}}\left|0_{\bar{\alpha}}\right\rangle=0, \quad \bar{\beta}_{\overrightarrow{\mathrm{p}}}\left|0_{\bar{\beta}}\right\rangle=0, \quad c_{n \overrightarrow{\mathrm{p}} \sigma}\left|0_{n}\right\rangle=0 .
$$

By using Eqs. (3.3) and (3.10) the transition matrix elements for the inelastic scattering $(i \neq f)$ corresponding to the transition $\alpha_{\frac{\mathrm{p}}{\mathrm{p}}}^{\dagger}\left|0_{\alpha n}\right\rangle \rightarrow \alpha_{\overrightarrow{\mathrm{p}}+\overrightarrow{\mathrm{q}}}^{\dagger}\left|0_{\alpha n}\right\rangle$ and $\beta_{\overrightarrow{\mathrm{p}}}^{\dagger}\left|0_{\beta n}\right\rangle \rightarrow \beta_{\overrightarrow{\mathrm{p}}+\overrightarrow{\mathrm{q}}}^{\dagger}\left|0_{\beta n}\right\rangle$ can be written

$$
\begin{aligned}
& \left\langle 0_{\alpha n}\left|\alpha_{\overrightarrow{\mathrm{p}}+\overrightarrow{\mathrm{q}}} t_{\mu \nu} \alpha \frac{\ddagger}{\mathrm{p}}\right| 0_{\alpha n}\right\rangle=S^{-1 / 2} \int_{z_{1}, z_{2}<0} d \overrightarrow{\mathrm{r}}_{1} d \overrightarrow{\mathrm{r}}_{2} e^{i\left(\left(\overrightarrow{\mathrm{k}}_{0} \mid \overrightarrow{\vec{r}}_{1}-\overrightarrow{\mathrm{k}}_{11} \overrightarrow{\vec{r}}_{2}\right)+\gamma_{0} z_{1}+\gamma_{s_{2}} z^{1}\right.}
\end{aligned}
$$

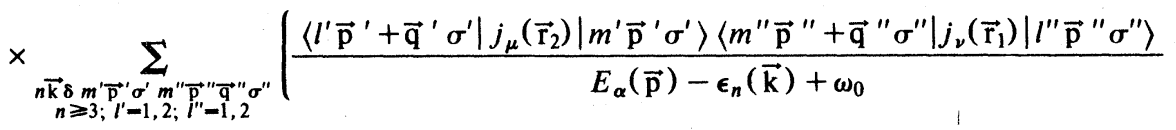

$$
\begin{aligned}
& \left.+\frac{\left\langle m^{\prime \prime} \overrightarrow{\mathrm{p}}^{\prime \prime}+\overrightarrow{\mathrm{q}}^{\prime \prime} \sigma^{\prime \prime}\left|j_{\nu}\left(\overrightarrow{\mathrm{r}}_{1}\right)\right| l^{\prime \prime} \overrightarrow{\mathrm{p}}^{\prime \prime} \sigma^{\prime \prime}\right\rangle\left\langle l^{\prime} \overrightarrow{\mathrm{p}}^{\prime}+\overrightarrow{\mathrm{q}}^{\prime} \sigma^{\prime}\left|j_{\mu}\left(\overrightarrow{\mathrm{r}}_{2}\right)\right| m^{\prime} \overrightarrow{\mathrm{p}}^{\prime} \sigma^{\prime}\right\rangle}{E_{\alpha}(\overrightarrow{\mathrm{p}})-\epsilon_{n}(\overrightarrow{\mathrm{k}})-\omega_{s}}\right) \\
& \times\left\langle 0_{\alpha}\left|\alpha_{\overrightarrow{\mathrm{p}}+\overrightarrow{\mathrm{q}}} c_{l^{\prime} \overrightarrow{\mathrm{p}}+\overrightarrow{\mathrm{q}}^{\prime} \sigma^{\prime}}^{\dagger} c_{l^{\prime \prime} \overrightarrow{\mathrm{p}}^{\prime \prime} \sigma^{\prime \prime}} \alpha_{\overrightarrow{\mathrm{p}}}^{\dagger}\right| 0_{\alpha}\right\rangle\left\langle 0_{n}\left|c_{m^{\prime} \overrightarrow{\mathrm{p}}^{\prime} \sigma^{\prime}} c_{n \overrightarrow{\mathrm{k} \delta}}^{\dagger}\right| 0_{n}\right\rangle \\
& \times\left\langle 0_{n}\left|c_{n \overrightarrow{\mathrm{k} \delta}} c_{m " \overrightarrow{\mathrm{p}}++\overrightarrow{\mathrm{q}} " \sigma^{\prime \prime}}^{\dagger}\right| 0_{n}\right\rangle,
\end{aligned}
$$


and

$$
\begin{aligned}
& \left\langle 0_{\beta n}\left|\beta_{\overrightarrow{\mathrm{p}}+\overrightarrow{\mathrm{q}}} t_{\mu \nu} \beta \frac{\dagger}{\mathrm{p}}\right| 0_{\beta n}\right\rangle=S^{-1 / 2} \int_{z_{1}, z_{2}<0} d \overrightarrow{\mathrm{r}}_{1} d \overrightarrow{\mathrm{r}}_{2} e^{i\left(\left(\overrightarrow{\mathrm{k}}_{011} \overrightarrow{\vec{r}}_{1}-\overrightarrow{-}_{\|}, \vec{F}_{2}\right)+\gamma_{0} z_{1}+\gamma_{s} z_{2}\right]}
\end{aligned}
$$

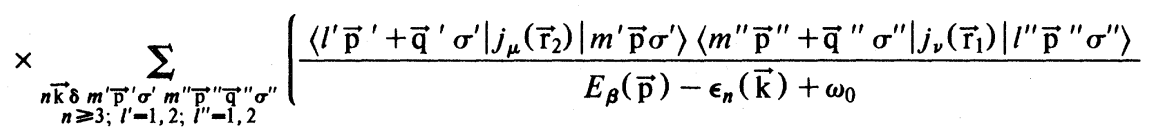

$$
\begin{aligned}
& \left.+\frac{\left\langle m^{\prime \prime} \overrightarrow{\mathrm{p}}^{\prime \prime}+\overrightarrow{\mathrm{q}}^{\prime \prime} \sigma^{\prime \prime}\left|j_{\nu}\left(\overrightarrow{\mathrm{r}}_{1}\right)\right| l^{\prime \prime} \overrightarrow{\mathrm{p}}^{\prime \prime} \sigma^{\prime \prime}\right\rangle\left\langle l^{\prime} \overrightarrow{\mathrm{p}}^{\prime}+\overrightarrow{\mathrm{q}}^{\prime} \sigma^{\prime}\left|j_{\mu}\left(\overrightarrow{\mathrm{r}}_{2}\right)\right| m^{\prime} \overrightarrow{\mathrm{p}}^{\prime} \sigma^{\prime}\right\rangle}{E_{\boldsymbol{\beta}}(\overrightarrow{\mathrm{p}})-\epsilon_{n}(\overrightarrow{\mathrm{k}})-\omega_{s}}\right) \\
& \times\left\langle 0_{\beta}\left|\beta_{\overrightarrow{\mathrm{p}}+\mathrm{q}^{\prime}} c_{l^{\prime} \overrightarrow{\mathrm{p}}^{\prime}+\overrightarrow{\mathrm{q}}^{\prime} \sigma^{\prime}}^{\dagger} c_{l^{\prime \prime} \overrightarrow{\mathrm{p}}^{\prime \prime} \sigma^{\prime \prime}} \boldsymbol{B}_{\overrightarrow{\mathrm{p}}}^{\dagger}\right| 0_{\beta}\right\rangle\left\langle 0_{n}\left|c_{m^{\prime} \overrightarrow{\mathrm{p}}^{\prime} \sigma^{\prime}} c_{n \overrightarrow{\mathrm{k} \delta}}^{\dagger}\right| 0_{n}\right\rangle \\
& \times\left\langle 0_{n}\left|c_{n \overrightarrow{\mathrm{k} \delta}} c_{m^{\prime \prime} \overrightarrow{\mathrm{p}}^{\prime \prime}+\vec{q}^{\prime \prime} \sigma^{\prime \prime}}\right| 0_{n}\right\rangle .
\end{aligned}
$$

By using Eqs. (2.5) and (4.5), the anticommutation relations for $\alpha$ 's, $\beta$ 's, $\bar{\alpha}$ 's, $\bar{\beta}$ 's, $c$ 's and summing over the possible spin configurations in Eqs. (4.6) and (4.7), one finds only the following terms different from zero:

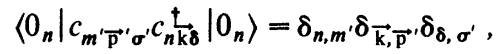

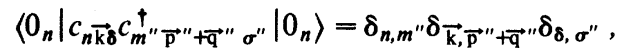

$$
\begin{aligned}
& \left\langle 0_{\alpha}\left|\alpha_{\vec{p}+\vec{q}^{\prime}} c_{1 \vec{p}^{\prime}+\vec{q}^{\prime} \mid}^{\dagger} c_{1 \vec{p}^{\prime \prime} \mid} \alpha_{\frac{\vec{p}}{\dagger}}^{\dagger}\right| 0_{\alpha}\right\rangle=u_{\vec{p}} u_{\vec{p}+\vec{q}} \delta_{\vec{p}+\vec{q}, \vec{p}^{\prime}+\vec{q}^{\prime}} \delta_{\vec{p}, \vec{p}^{\prime \prime}},
\end{aligned}
$$

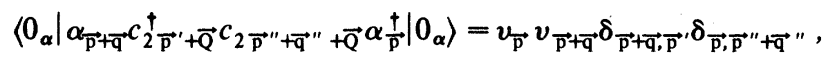

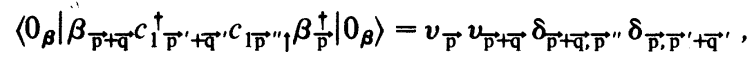

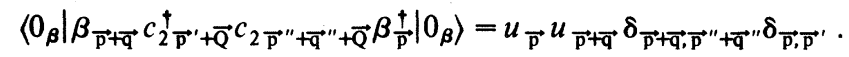

At this state we assume that the following approximations are valid:

$$
\begin{aligned}
& \left\langle m \overrightarrow{\mathrm{p}}+\overrightarrow{\mathrm{q}} \sigma\left|j_{\mu}(\overrightarrow{\mathrm{r}})\right| n \overrightarrow{\mathrm{p}} \sigma\right\rangle=\left\langle m \overrightarrow{\mathrm{p}}+\overrightarrow{\mathrm{Q}}+\overrightarrow{\mathrm{q}} \sigma\left|j_{\mu}(\overrightarrow{\mathrm{r}})\right| n \overrightarrow{\mathrm{p}}+\overrightarrow{\mathrm{Q}} \sigma\right\rangle=\left\langle c \overrightarrow{\mathrm{p}}\left|j_{\mu}(\overrightarrow{\mathrm{r}})\right| n \overrightarrow{\mathrm{p}}\right\rangle, n \geqslant 3, \quad m=1,2, \\
& \epsilon_{n}(\overrightarrow{\mathrm{p}}) \simeq \epsilon_{n}(\overrightarrow{\mathrm{p}}+Q) .
\end{aligned}
$$

Then with Eqs. (4.8), (4.9), (2.11), and (2.12), and after performing the summations in Eqs. (4.6) and (4.7) one obtains the following expression for the transiition matrix elements:

$$
\begin{aligned}
& \left\langle 0_{\alpha n}\left|\alpha_{\overrightarrow{\mathrm{p}}+\mathrm{q}} t_{\mu \nu} \alpha \frac{\dagger}{\dagger}\right| 0_{\alpha n}\right\rangle=M_{\mu \nu}^{N}\left(\overrightarrow{\mathrm{p}}, \omega_{0}, \omega_{s} ; \alpha\right) l(\overrightarrow{\mathrm{p}}, \overrightarrow{\mathrm{q}}), \\
& \left\langle 0_{\beta n}\left|\beta_{\overrightarrow{\mathrm{p}}+\overrightarrow{\mathrm{q}}} t_{\mu \nu} \beta \frac{\dagger}{\mathrm{p}}\right| 0_{\beta n}\right\rangle=M_{\mu \nu}^{N}\left(\overrightarrow{\mathrm{p}}, \omega_{0}, \omega_{s} ; \beta\right) l(\overrightarrow{\mathrm{p}}, \overrightarrow{\mathrm{q}}),
\end{aligned}
$$

where

$$
\begin{aligned}
& M_{\mu \nu}^{N}\left(\overrightarrow{\mathrm{p}}, \omega_{0}, \omega_{s} ; \alpha\right)=S^{-1 / 2} \int_{z_{1}, z_{2}<0} d \overrightarrow{\mathrm{r}}_{1} d \overrightarrow{\mathrm{r}}_{2} e^{\left.i\left(\overrightarrow{\mathrm{k}}_{011_{1}}-\overrightarrow{\mathrm{r}}_{1} \overrightarrow{\mathrm{r}}_{2}\right)+\gamma_{0} \mathrm{z}_{1}+\gamma_{s} \mathbf{z}_{2}\right]} \\
& \times \sum_{n \geqslant 3}\left(\frac{\left\langle c \overrightarrow{\mathrm{p}}\left|j_{\mu}\left(\overrightarrow{\mathrm{r}}_{2}\right)\right| n \overrightarrow{\mathrm{p}}\right\rangle\left\langle n \overrightarrow{\mathrm{p}}\left|j_{\nu}\left(\overrightarrow{\mathrm{r}}_{1}\right)\right| c \overrightarrow{\mathrm{p}}\right\rangle}{E_{\alpha}(\overrightarrow{\mathrm{p}})-\epsilon_{n}(\overrightarrow{\mathrm{p}})+\omega_{0}}+\frac{\left\langle c \overrightarrow{\mathrm{p}}\left|j_{\nu}\left(\overrightarrow{\mathrm{r}}_{1}\right)\right| n \overrightarrow{\mathrm{p}}\right\rangle\left\langle n \overrightarrow{\mathrm{p}}\left|j_{\mu}\left(\overrightarrow{\mathrm{r}}_{2}\right)\right| c \overrightarrow{\mathrm{p}}\right\rangle}{E_{\alpha}(\overrightarrow{\mathrm{p}})-\epsilon_{n}(\overrightarrow{\mathrm{p}})-\omega_{s}}\right), \\
& M_{\mu \nu}^{N}\left(\overrightarrow{\mathrm{p}}, \omega_{0}, \omega_{s} ; \beta\right)=S^{-1 / 2} \int_{z_{1}, z_{2}<0} d \overrightarrow{\mathrm{r}}_{1} d \overrightarrow{\mathrm{r}}_{2} e^{i\left[\left(\overrightarrow{\mathrm{k}}_{011} \overrightarrow{\mathrm{r}}_{1}-\overrightarrow{\mathrm{k}}_{1} \overrightarrow{\vec{r}}_{2}\right)+\gamma_{0} z_{1}+\gamma_{s} z_{2}\right]} \\
& \times \sum_{n \geqslant 3}\left(\frac{\left\langle c \overrightarrow{\mathrm{p}}\left|j_{\mu}\left(\overrightarrow{\mathrm{r}}_{2}\right)\right| n \overrightarrow{\mathrm{p}}\right\rangle\left\langle n \overrightarrow{\mathrm{p}}\left|j_{\nu}\left(\overrightarrow{\mathrm{r}}_{1}\right)\right| c \overrightarrow{\mathrm{p}}\right\rangle}{E_{\boldsymbol{\beta}}(\overrightarrow{\mathrm{p}})-\epsilon_{n}(\overrightarrow{\mathrm{p}})+\omega_{0}}+\frac{\left\langle c \overrightarrow{\mathrm{p}}\left|j_{\nu}\left(\overrightarrow{\mathrm{r}}_{1}\right)\right| n \overrightarrow{\mathrm{p}}\right\rangle\left\langle n \overrightarrow{\mathrm{p}}\left|j_{\mu}\left(\overrightarrow{\mathrm{r}}_{2}\right)\right| c \overrightarrow{\mathrm{p}}\right\rangle}{E_{\beta}(\overrightarrow{\mathrm{p}})-\epsilon_{n}(\overrightarrow{\mathrm{p}})-\omega_{s}}\right),
\end{aligned}
$$


and

$$
l(\overrightarrow{\mathrm{p}}, \overrightarrow{\mathrm{q}})=u_{\overrightarrow{\mathrm{p}}} u_{\overrightarrow{\mathrm{p}}+\overrightarrow{\mathrm{q}}}+v_{\overrightarrow{\mathrm{p}}} v_{\overrightarrow{\mathrm{p}}+\overrightarrow{\mathrm{q}}}=\frac{1}{\sqrt{2}}\left(1+\frac{\epsilon_{12}(\overrightarrow{\mathrm{p}}) \epsilon_{12}(\overrightarrow{\mathrm{p}}+\overrightarrow{\mathrm{q}})+4 g^{2}}{E_{\alpha \beta}(\overrightarrow{\mathrm{p}}) E_{\alpha \beta}(\overrightarrow{\mathrm{p}}+\overrightarrow{\mathrm{q}})}\right)^{1 / 2},
$$

is the usual coherence factor and the following abbreviations were introduced:

$$
\epsilon_{12}(\overrightarrow{\mathrm{p}}) \equiv \epsilon_{1}(\overrightarrow{\mathrm{p}})-\epsilon_{2}(\overrightarrow{\mathrm{p}}+\overrightarrow{\mathrm{Q}}), \quad E_{\alpha \beta}(\overrightarrow{\mathrm{p}}) \equiv E_{\alpha}(\overrightarrow{\mathrm{p}})-E_{\beta}(\overrightarrow{\mathrm{p}})
$$

Transitions $\bar{\alpha}_{-\overrightarrow{\mathrm{p}}-\overrightarrow{\mathrm{Q}}}^{\dagger}\left|0_{\bar{\alpha} n}\right\rangle \rightarrow \bar{\alpha}_{-\overrightarrow{\mathrm{p}}-\overrightarrow{\mathrm{Q}}-\overrightarrow{\mathrm{q}}}^{\dagger}\left|0_{\bar{\alpha} n}\right\rangle$ and $\bar{\beta}_{-\overrightarrow{\mathrm{p}}-\overrightarrow{\mathrm{Q}}}^{\dagger}\left|0_{\beta n}\right\rangle \rightarrow \bar{\beta}_{-\overrightarrow{\mathrm{p}}-\overrightarrow{\mathrm{Q}}-\overrightarrow{\mathrm{q}}}^{\dagger}\left|0_{\bar{\beta} n}\right\rangle$ lead to identical transition matrix elements given by Eqs. (4.10) and (4.11), respectively, i.e.,

$$
\begin{aligned}
& \left|\left\langle 0_{\alpha n}\left|\alpha_{\overrightarrow{\mathrm{p}}+\vec{q}} t_{\mu \nu} \alpha_{\overrightarrow{\mathrm{p}}}^{\dagger}\right| 0_{\alpha n}\right\rangle\right|=\left|\left\langle 0_{\bar{\alpha} n}\left|\bar{\alpha}_{-\overrightarrow{\mathrm{p}}-\overrightarrow{\mathrm{Q}}-\overrightarrow{\mathrm{q}}} t_{\mu \nu} \bar{\alpha}_{-\overrightarrow{\mathrm{p}}-\overrightarrow{\mathrm{Q}}}^{\dagger}\right| 0_{\alpha n}\right\rangle\right|, \\
& \left|\left\langle 0_{\beta n}\left|\beta_{\overrightarrow{\mathrm{p}}+\overrightarrow{\mathrm{q}}} t_{\mu \nu} \beta_{\overrightarrow{\mathrm{p}}}^{\dagger}\right| 0_{\beta n}\right\rangle\right|=\left|\left\langle 0_{\bar{\beta} n}\left|\bar{\beta}_{-\overrightarrow{\mathrm{p}}-\overrightarrow{\mathrm{Q}}-\overrightarrow{\mathrm{q}}} t_{\mu \nu} \bar{\beta}_{-\overrightarrow{\mathrm{p}}-\overrightarrow{\mathrm{Q}}}^{\dagger}\right| 0_{\bar{\beta} n}\right\rangle\right| .
\end{aligned}
$$

With Eqs. (4.1)-(4.3), (4.10), (4.11), (4.16), and (4.17) the scattering efficiency for the inelastic scattering of light by the normal fluid one-particle excitations in antiferromagnetic chromium can be written in the form

$$
\frac{1}{\phi_{0}}\left(\frac{d^{2} \phi}{d \omega d \Omega}\right)_{N}=\frac{1}{\phi_{0}}\left(\frac{d^{2} \phi}{d \omega d \Omega}\right)_{N \alpha}+\frac{1}{\phi_{0}}\left(\frac{d^{2} \phi}{d \omega d \Omega}\right)_{N \beta},
$$

where

$$
\begin{aligned}
\frac{1}{\phi_{0}}\left(\frac{d^{2} \phi}{d \omega d \Omega}\right)_{N \alpha}=\frac{4 \omega_{s} \cos ^{2} \theta_{s}}{S \omega_{0} c^{4} \cos \theta_{0}} \sum_{\overrightarrow{\mathrm{p}} \in A_{m}} \sum_{\substack{\mu \nu \eta^{\prime} \nu^{\prime} \eta^{\prime} \xi^{\prime} \\
\mu_{s \mu}}} e_{s \nu} e_{\mu \mu^{\prime}}\left(\overrightarrow{\mathrm{k}}_{\|}, \omega_{s}\right) D_{\nu \nu^{\prime}}^{*}\left(\overrightarrow{\mathrm{k}}_{\| 1}, \omega_{s}\right) M_{\mu^{\prime} \eta^{\prime}}^{N}\left(\overrightarrow{\mathrm{p}}, \omega_{0}, \omega_{s} ; \alpha\right) \\
\times M_{\nu^{\prime} \xi^{\prime}}^{N^{*}}\left(\overrightarrow{\mathrm{p}}, \omega_{0}, \omega_{s} ; \alpha\right) T_{\eta^{\prime} \eta} T_{\xi^{\prime} \xi}^{*} e_{0 \eta} e_{0 \xi} l^{2}(\overrightarrow{\mathrm{p}}, \overrightarrow{\mathrm{q}}) \\
\times\left[f\left(E_{\alpha}(\overrightarrow{\mathrm{p}})\right)-f\left(E_{\alpha}(\overrightarrow{\mathrm{p}}+\overrightarrow{\mathrm{q}})\right)\right] \delta\left(\omega+E_{\alpha}(\overrightarrow{\mathrm{p}})-E_{\alpha}(\overrightarrow{\mathrm{p}}+\overrightarrow{\mathrm{q}})\right),
\end{aligned}
$$

and

$$
\begin{aligned}
\frac{1}{\phi_{0}}\left(\frac{d^{2} \phi}{d \omega d \Omega}\right)_{N \beta}=\frac{4 \omega_{s} \cos ^{2} \theta_{s}}{S \omega_{0} c^{4} \cos \theta_{0}} \sum_{\overrightarrow{\mathrm{p}} \in A_{m}} \sum_{\substack{\mu \nu, \eta \xi^{\prime} \\
\mu^{\prime} \nu^{\prime} \eta^{\prime} \xi^{\prime}}} e_{s \mu} e_{s \nu} D_{\mu \mu^{\prime}}\left(\overrightarrow{\mathrm{k}}_{\|,}, \omega_{s}\right) D_{\nu \nu^{\prime}}^{*}\left(\overrightarrow{\mathrm{k}}_{\|,}, \omega_{s}\right) M_{\mu^{\prime} \eta^{\prime}}^{N}\left(\overrightarrow{\mathrm{p}}, \omega_{0}, \omega_{s} ; \beta\right) \\
\times M_{v^{\prime} \xi^{\prime}}^{N^{*}}\left(\overrightarrow{\mathrm{p}}, \omega_{0}, \omega_{s} ; \beta\right) T_{\eta^{\prime} \eta} T_{\xi^{\prime} \xi}^{*} e_{0 \eta} e_{0 \xi^{\prime}} l^{2}(\overrightarrow{\mathrm{p}}, \overrightarrow{\mathrm{q}}) \\
\times\left[f\left(E_{\beta}(\overrightarrow{\mathrm{p}}+\overrightarrow{\mathrm{q}})\right)-f\left(E_{\beta}(\overrightarrow{\mathrm{p}})\right)\right] \delta\left(\omega+E_{\beta}(\overrightarrow{\mathrm{p}}+\overrightarrow{\mathrm{q}})-E_{\beta}(\overrightarrow{\mathrm{p}})\right)
\end{aligned}
$$

From Eqs. (4.19) and (4.20) we see that the scattering can occur only at $T \neq 0^{\circ} \mathrm{K}$.

The scattering processes which lead to the appearance of coherence factors in Eqs. (4.10) and (4.11) are shown schematically in Fig. 2. Figures 2 (a) and 2 (c) are the two configurations entering the wave function for a state with quasiparticle in $1 \vec{p} \uparrow$. Figures 2(b) and 2(d) are the two configurations which can be connected to Figs. 2(a) and 2(c), respectively, by a spin independent one-body operator. The arrows indicate how Figs. 2 (a) $\rightarrow 2$ (b) and 2 (c) $\rightarrow 2$ (d) via intermediate interband states $n$, when electrons couple to the electromagnetic field which does not flip the electronic spin.

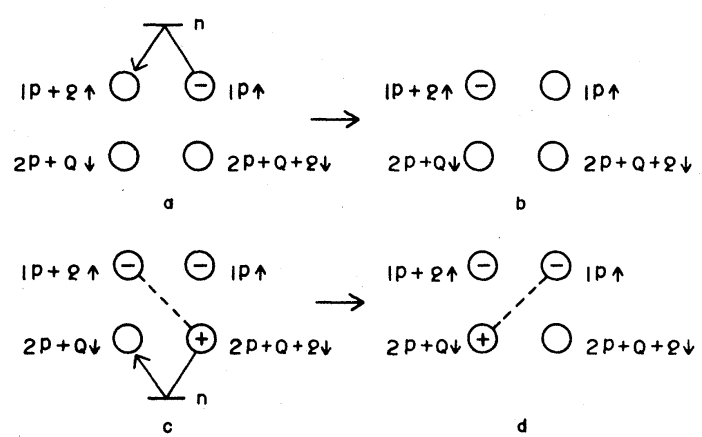

FIG. 2. Schematic representation of the inelastic scattering of light in antiferromagnetic chromium by one-electron and one-hole excitations in the temperature interval $T_{N}>T>0^{\circ} \mathrm{K}$. 


\section{SCATTERING OF LIGHT BY TWO-PARTICLE EXCITATIONS}

The "magnetic" scattering cross section involves the breaking of bound-electron-hole pairs in triplet state and the creation of pairs of quasiparticles with minimum excitaiton energy being $2 \mathrm{~g}$. One can distinguish the following initial and final states, corresponding initial- and final-state excitation energies and thermal-weight rate factors:

$$
\begin{aligned}
& (|i\rangle,|f\rangle)=\left(\left|0_{\alpha \beta n}\right\rangle, \alpha \frac{\bar{p}+\vec{q} \beta}{\dagger} \beta_{\bar{p}}^{\dagger}\left|0_{\alpha \beta n}\right\rangle\right), \quad\left(\left|0_{\bar{\alpha} \bar{\beta} n}\right\rangle, \bar{\alpha}_{-\vec{p}-\overrightarrow{\mathrm{Q}}-\overrightarrow{\mathrm{q}}}^{\dagger} \bar{\beta}_{-\overrightarrow{\mathrm{p}}-\overrightarrow{\mathrm{Q}}}^{\dagger}\left|0_{\bar{\alpha} \bar{\beta} n}\right\rangle\right), \\
& \left(E_{i}, E_{f}\right)=\left(0, E_{\alpha}(\overrightarrow{\mathrm{p}}+\overrightarrow{\mathrm{q}})-E_{\beta}(\overrightarrow{\mathrm{p}})\right), \quad\left(0, E_{\alpha}(\overrightarrow{\mathrm{p}}+\overrightarrow{\mathrm{q}})-E_{\beta}(\overrightarrow{\mathrm{p}})\right), \\
& \left(P_{i}-P_{f}\right)=\left[f\left(E_{\beta}(\overrightarrow{\mathrm{p}})\right)-f\left(E_{\alpha}(\overrightarrow{\mathrm{p}}+\overrightarrow{\mathrm{q}})\right)\right], \quad\left[f\left(E_{\beta}(\overrightarrow{\mathrm{p}})\right)-f\left(E_{\alpha}(\overrightarrow{\mathrm{p}}+\overrightarrow{\mathrm{q}})\right)\right], \\
& \left|0_{\alpha \beta n}\right\rangle \equiv\left|0_{\alpha}\right\rangle\left|0_{\beta}\right\rangle\left|0_{n}\right\rangle, \quad\left|0_{\bar{\alpha} \bar{\beta} n}\right\rangle \equiv\left|0_{\bar{\alpha}}\right\rangle\left|0_{\bar{\beta}}\right\rangle\left|0_{n}\right\rangle .
\end{aligned}
$$

In above equations the relations given by Eqs. (2.7) and (2.8) were taken into account.

By using Eqs. (3.3) and (3.10) the transition matrix elements for the inelastic scattering $(i \neq f)$ corresponding to the transitions $\left|0_{\alpha \beta n}\right\rangle \rightarrow \alpha_{\overline{\mathrm{p}}+\overrightarrow{\mathrm{q}}}^{\dagger} \beta_{\overrightarrow{\mathrm{p}}}^{\dagger}\left|0_{\alpha \beta n}\right\rangle$ and $\left|0_{\bar{\alpha} \bar{\beta} n}\right\rangle \rightarrow \bar{\alpha}_{-\overrightarrow{\mathrm{p}}-\overrightarrow{\mathrm{Q}}-\overline{\mathrm{q}}}^{\dagger} \bar{\beta}_{-\overrightarrow{\mathrm{p}}-\overrightarrow{\mathrm{Q}}}^{\dagger}\left|0_{\bar{\alpha} \bar{\beta} n}\right\rangle$ can be written

$$
\begin{aligned}
& \left\langle 0_{\alpha \beta n}\left|\beta_{\overrightarrow{\mathrm{p}}} \alpha_{\overrightarrow{\mathrm{p}}+\vec{q}} t_{\mu \nu}\right| 0_{\alpha \beta n}\right\rangle=S^{-1 / 2} \int_{z_{1}, z_{2}<0} d \overrightarrow{\mathrm{r}}_{1} d \overrightarrow{\mathrm{r}}_{2} e^{i\left(\left(\overrightarrow{\mathrm{k}}_{0,1} \overrightarrow{\mathrm{r}}_{1}-\overrightarrow{\mathrm{k}}_{1} \overrightarrow{\mathrm{r}}_{2}\right)+\gamma_{0} \mathrm{z}_{1}+\gamma_{s} \mathrm{z}_{2}\right]}
\end{aligned}
$$

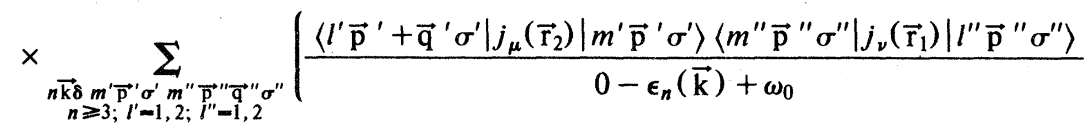

$$
\begin{aligned}
& \left.+\frac{\left\langle m^{\prime \prime} \overrightarrow{\mathrm{p}}^{\prime \prime}+\overrightarrow{\mathrm{q}}^{\prime \prime}\left|j_{\nu}\left(\overrightarrow{\mathrm{r}}_{1}\right)\right| l^{\prime \prime} \overrightarrow{\mathrm{p}}^{\prime \prime} \sigma^{\prime \prime}\right\rangle\left\langle l^{\prime} \overrightarrow{\mathrm{p}}^{\prime}+\overrightarrow{\mathrm{q}}^{\prime} \sigma^{\prime}\left|j_{\mu}\left(\overrightarrow{\mathrm{r}}_{2}\right)\right| m^{\prime} \overrightarrow{\mathrm{p}}^{\prime} \sigma^{\prime}\right\rangle}{0-\epsilon_{n}(\overrightarrow{\mathrm{k}})-\omega_{s}}\right) \\
& \times\left\langle 0_{\alpha \beta}\left|\beta_{\overrightarrow{\mathrm{p}}} \alpha_{\overrightarrow{\mathrm{p}}+\overrightarrow{\mathrm{q}}} c_{l^{\prime} \overrightarrow{\mathrm{p}}^{\prime}+\mathrm{q}^{\prime} \sigma^{\prime}}^{\dagger} c_{l^{\prime \prime} \overrightarrow{\mathrm{p}}^{\prime \prime} \sigma^{\prime \prime}}\right| 0_{\alpha \beta}\right\rangle \\
& \times\left\langle 0_{n}\left|c_{m^{\prime} \overrightarrow{\mathrm{p}}^{\prime} \sigma^{\prime}} c_{n \overrightarrow{\mathrm{k} \delta}}^{\dagger}\right| 0_{n}\right\rangle\left\langle 0_{n}\left|c_{n \overrightarrow{\mathrm{k} \delta}} c_{m^{\prime \prime} \overrightarrow{\mathrm{p}}^{\prime \prime}+\overrightarrow{\mathrm{q}}^{\prime \prime} \sigma^{\prime \prime}}^{\dagger}\right| 0_{n}\right\rangle
\end{aligned}
$$

and

$$
\begin{aligned}
& \left\langle 0_{\bar{\alpha} \bar{\beta} n}\left|\bar{\beta}_{-\overrightarrow{\mathrm{p}}-\overrightarrow{\mathrm{Q}}} \bar{\alpha}_{-\overrightarrow{\mathrm{p}}-\overrightarrow{\mathrm{Q}}+\overrightarrow{\mathrm{Q}}} t_{\mu \nu}\right| 0_{\bar{\alpha} \bar{\beta} n}\right\rangle=S^{-1 / 2} \int_{z_{1}, z_{2}<0} d \overrightarrow{\mathrm{r}}_{1} d \overrightarrow{\mathrm{r}}_{2} e^{i\left(\left(\overrightarrow{\mathrm{k}}_{01 \mathrm{r}_{1}}-\overrightarrow{\mathrm{k}}_{1} \overrightarrow{\vec{r}}_{2}\right)+\gamma_{0} z_{1}+\gamma_{s} z_{2}\right]}
\end{aligned}
$$

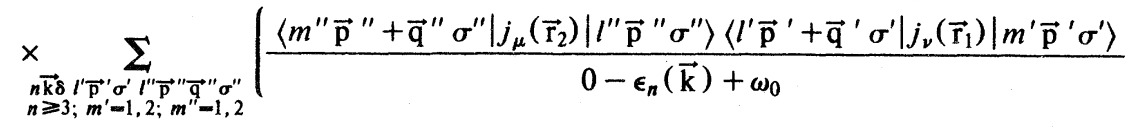

$$
\begin{aligned}
& \left.+\frac{\left\langle m^{\prime \prime} \overrightarrow{\mathrm{p}}^{\prime \prime} \sigma^{\prime \prime}\left|j_{\nu}\left(\overrightarrow{\mathrm{r}}_{1}\right)\right| l^{\prime \prime} \overrightarrow{\mathrm{p}}^{\prime \prime} \sigma^{\prime \prime}\right\rangle\left\langle l^{\prime} \overrightarrow{\mathrm{p}}^{\prime}+\overrightarrow{\mathrm{q}}^{\prime} \sigma^{\prime}\left|j_{\mu}\left(\overrightarrow{\mathrm{r}}_{2}\right)\right| m^{\prime} \overrightarrow{\mathrm{p}}^{\prime} \sigma^{\prime}\right\rangle}{0-\epsilon_{n}(\overrightarrow{\mathrm{k}})-\omega_{s}}\right) \\
& \times\left\langle 0_{\bar{\alpha} \bar{\beta}}\left|\bar{\beta}_{-\vec{p}-\vec{Q}} \bar{\alpha}_{-\vec{p}-\vec{Q}-\vec{q}} c_{l^{\prime} \vec{p}^{\prime}+\vec{q}^{\prime} \sigma^{\prime \prime}}^{\dagger} c_{l " \vec{p}^{\prime \prime} \sigma^{\prime \prime}}\right| 0_{\alpha \beta}\right\rangle \\
& \times\left\langle 0_{n}\left|c_{n^{\prime} \overrightarrow{\mathrm{p}}^{\prime} \boldsymbol{\sigma}^{\prime}} c_{n \mathrm{k} \mathbf{k}}^{\dagger}\right| 0_{n}\right\rangle\left\langle 0_{n}\left|c_{n \overrightarrow{\mathrm{k} \delta}} c_{m^{\prime \prime} \overrightarrow{\mathrm{p}} "+\overrightarrow{\mathrm{q}}^{\prime \prime} \sigma^{\prime \prime}}^{\dagger}\right| 0_{n}\right\rangle .
\end{aligned}
$$

By using Eqs. (2.5) and (4.5), the anticommutation relations for $\alpha$ 's, $\beta$ 's, $\bar{\alpha}$ 's, $\bar{\beta}$ 's, and $c$ 's and summing over the possible spin configurations in Eqs. (5.4) and (5.5), one finds only the following terms different from zero:

$$
\begin{aligned}
& \left\langle 0_{n}\left|c_{m^{\prime} \overrightarrow{\mathrm{p}}^{\prime} \sigma^{\prime}} c_{n \overrightarrow{\mathrm{k} \delta}}^{t}\right| 0_{n}\right\rangle=\delta_{n, m^{\prime}} \delta_{\overrightarrow{\mathrm{k}}, \overrightarrow{\mathrm{p}}^{\prime}}, \delta_{\delta, \sigma^{\prime}},
\end{aligned}
$$

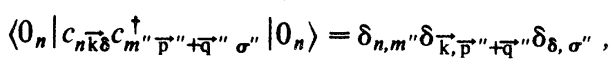

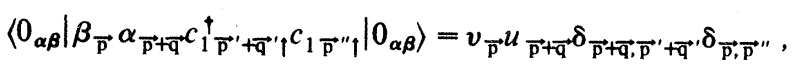

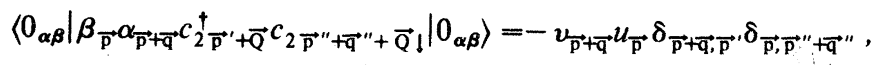

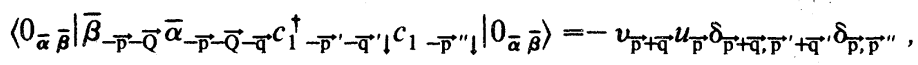

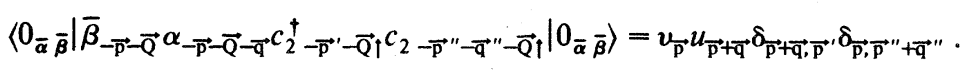


With Eqs. (4.9), (5.6), (2.11), and (2.12), and after performing the summations in Eqs. (5.4) and (5.5), one obtains the following expressions for the transition matrix elements:

$$
\begin{aligned}
& \left\langle 0_{\alpha \beta n}\left|\beta_{\overrightarrow{\mathrm{p}}} \alpha_{\overrightarrow{\mathrm{p}}+\vec{q}} t_{\mu \nu}\right| 0_{\alpha \beta n}\right\rangle=M_{\mu \nu}^{M}\left(\overrightarrow{\mathrm{p}}, \omega_{0}, \omega_{s} ; \alpha, \beta\right) p(\overrightarrow{\mathrm{p}}, \overrightarrow{\mathrm{q}}), \\
& \left\langle 0_{\bar{\alpha} \bar{\beta} n}\left|\bar{\beta}_{-\overrightarrow{\mathrm{p}}-\overrightarrow{\mathrm{Q}}} \bar{\alpha}_{-\overrightarrow{\mathrm{p}}-\overrightarrow{\mathrm{Q}}-\overrightarrow{\mathrm{q}}} t_{\mu \nu}\right| 0_{\bar{\alpha} \bar{\beta} n}\right\rangle=\left\langle 0_{\alpha \beta n}\left|\beta_{\overrightarrow{\mathrm{p}}} \alpha_{\overrightarrow{\mathrm{p}}+\vec{q}} t_{\mu \nu}\right| 0_{\alpha \beta n}\right\rangle,
\end{aligned}
$$

where

$$
\begin{array}{r}
M_{\mu \nu}^{M}\left(\overrightarrow{\mathrm{p}}, \omega_{0}, \omega_{s} ; \alpha \beta\right)=S^{-1 / 2} \int_{z_{1}, z_{2}<0} d \overrightarrow{\mathrm{r}}_{1} d \overrightarrow{\mathrm{r}}_{2} e^{i\left(\left(\overrightarrow{\mathrm{k}}_{011} \overrightarrow{\mathrm{r}}_{1}-\overrightarrow{\mathrm{k}}_{11} \overrightarrow{\vec{r}}_{2}\right)+\gamma_{0} z_{1}+\gamma_{s} z_{2}\right]} \sum_{n \geqslant 3}\left(\frac{\left\langle c \overrightarrow{\mathrm{p}}\left|j_{\mu}\left(\overrightarrow{\mathrm{r}}_{2}\right)\right| n \overrightarrow{\mathrm{p}}\right\rangle\left\langle n \overrightarrow{\mathrm{p}}\left|j_{\nu}\left(\overrightarrow{\mathrm{r}}_{1}\right)\right| c \overrightarrow{\mathrm{p}}\right\rangle}{-\epsilon_{n}(\overrightarrow{\mathrm{p}})+\omega_{0}}\right. \\
\left.+\frac{\left\langle c \overrightarrow{\mathrm{p}}\left|j_{\nu}\left(\overrightarrow{\mathrm{r}}_{1}\right)\right| n \overrightarrow{\mathrm{p}}\right\rangle\left\langle n \overrightarrow{\mathrm{p}}\left|j_{\mu}\left(\overrightarrow{\mathrm{r}}_{2}\right)\right| c \overrightarrow{\mathrm{p}}\right\rangle}{-\epsilon_{n}(\overrightarrow{\mathrm{p}})-\omega_{s}}\right)
\end{array}
$$

and $p(\overrightarrow{\mathrm{p}}, \overrightarrow{\mathrm{q}})$ is the coherence factor, given by

$$
p(\overrightarrow{\mathrm{p}}, \overrightarrow{\mathrm{q}})=v_{\overrightarrow{\mathrm{p}}} u_{\overrightarrow{\mathrm{p}}+\overrightarrow{\mathrm{q}}}-v_{\overrightarrow{\mathrm{p}}+\overrightarrow{\mathrm{q}}} u_{\overrightarrow{\mathrm{p}}}=\frac{1}{\sqrt{2}}\left(1-\frac{\epsilon_{12}(\overrightarrow{\mathrm{p}}) \epsilon_{12}(\overrightarrow{\mathrm{p}}+\overrightarrow{\mathrm{q}})+4 g^{2}}{E_{\alpha \beta}(\overrightarrow{\mathrm{p}}) E_{\alpha \beta}(\overrightarrow{\mathrm{p}}+\overrightarrow{\mathrm{q}})}\right)^{1 / 2} .
$$

With Eqs. (5.1) - (5.3), (5.7), and (5.8) the scattering efficiency for the inelastic scattering of light by the magnetic two-particle excitations in antiferromagnetic chromium can be written in the form

$$
\begin{aligned}
& \frac{1}{\phi_{0}}\left(\frac{d^{2} \phi}{d \omega d \Omega}\right)_{M}=\frac{4 \omega_{s} \cos ^{2} \theta_{s}}{S \omega_{0} c^{4} \cos \theta_{0}} \sum_{\overrightarrow{\mathrm{p}} \in A_{m}} \sum_{\substack{\mu \nu \eta^{\prime} \xi \\
\mu^{\prime} \nu^{\prime} \eta^{\prime} \xi^{\prime}}} e_{s \mu} e_{s \nu} D_{\mu \mu^{\prime}}\left(\overrightarrow{\mathrm{k}}_{\|}, \omega_{s}\right) D_{\nu \nu^{\prime}}^{*}\left(\overrightarrow{\mathrm{k}}_{\|}, \omega_{s}\right) M_{\mu^{\prime} \eta^{\prime}}^{M}\left(\overrightarrow{\mathrm{p}}, \omega_{0}, \omega_{s} ; \alpha, \beta\right) \\
& \times M_{\nu \xi^{\prime}}^{M^{*}}\left(\overrightarrow{\mathrm{p}}, \omega_{0}, \omega_{s} ; \alpha, \beta\right) T_{\eta^{\prime} \eta} T_{\xi^{\prime} \xi}^{*} e_{0 \eta} e_{0 \xi} p^{2}(\overrightarrow{\mathrm{p}}, \overrightarrow{\mathrm{q}}) \\
& \times\left[f\left(E_{\beta}(\overrightarrow{\mathrm{p}})\right)-f\left(E_{\alpha}(\overrightarrow{\mathrm{p}}+\overrightarrow{\mathrm{q}})\right)\right] \delta\left(\omega-E_{\alpha}(\overrightarrow{\mathrm{p}}+\overrightarrow{\mathrm{q}})+E_{\beta}(\overrightarrow{\mathrm{p}})\right)
\end{aligned}
$$

From Eq. (5.11) we see that the scattering is possible even at $T=0^{\circ} \mathrm{K}$ since $E_{\beta}(\overrightarrow{\mathrm{p}})<0$.

The scattering processes which lead to the appearance of $p$ coherence factor in Eq. (5.7) are shown schematically in Fig. 3. Figures $3(\mathrm{a})$ and 3(b) are the only two configurations which can be connected to that in Fig. 3(c) by a spin-independent one-body operator. The arrows indicate how Figs. $3(\mathrm{a}) \rightarrow 3(\mathrm{c})$

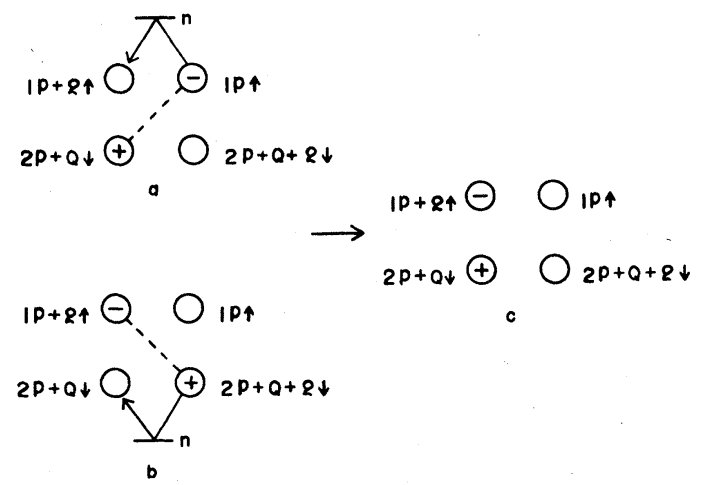

FIG. 3. Schematic representation of the inelastic scattering of light in antiferromagnetic chromium by one-electron pair in triplet state in the temperature interval $T_{N}>T \geqslant 0^{\circ} \mathrm{K}$. and $3(\mathrm{~b}) \rightarrow 3$ (c) via intermediate interband states $n$, when electrons couple to the electromagnetic field which does not flip the spin.

\section{TOTAL SCATTERING EFFICIENCY}

Clearly, the total scattering efficiency is the sum

$$
\begin{aligned}
& \frac{1}{\phi_{0}}\left(\frac{d^{2} \phi}{d \omega d \Omega}\right) \\
& =\frac{1}{\phi_{0}}\left(\frac{d^{2} \phi}{d \omega d \Omega}\right)_{n}+\frac{1}{\phi_{0}}\left(\frac{d^{2} \phi}{d \omega d \Omega}\right)_{N}+\frac{1}{\phi_{0}}\left(\frac{d^{2} \phi}{d \omega d \Omega}\right)_{M}
\end{aligned}
$$

The normal-metal scattering efficiency can be obtained simply by putting $g=0$ and substituting $A_{m} \rightarrow A$ for $T>T_{N}$, and $A_{m} \rightarrow A_{n}$ for $T<T_{N}$ in Eq. (4.18).

From Eqs. (4.12), (4.13), and (5.9) it is clear that the important intermediate states are the nonconducting states for which an energy denominator is small.

For further calculations one has to specify the momentum dependence of band energies and matrix elements. For simplicity, let us assume that near the magnetic portion of Fermi surface with area $A_{m}$ over 
which the gap forms one can write approximately

$$
\begin{aligned}
& \epsilon_{12} \equiv \epsilon_{1}(\vec{p})-\epsilon_{2}(\vec{p}+\vec{Q}) \simeq \epsilon_{1}(\vec{p})\left(1-\frac{\nabla_{\vec{p}} \epsilon_{2}(\vec{p}+\vec{Q})}{\nabla_{\vec{p}} \epsilon_{1}(\vec{p})}\right) \simeq a \epsilon_{1}(\vec{p}) \\
& \epsilon_{1}(\vec{p})+\epsilon_{2}(\vec{p}+\vec{Q}) \simeq \epsilon_{1}(\vec{p})\left(1+\frac{\nabla_{\vec{p}} \epsilon_{2}(\vec{p}+\vec{Q})}{\nabla_{\vec{p}} \epsilon_{1}(\vec{p})}\right) \simeq b \epsilon_{1}(\vec{p})
\end{aligned}
$$

where $a$ and $b$ represent some appropriate average value of the bracketed quantities.

If the energy band 1 is assumed parabolic, then for conditions of large momentum transfer, i.e., $v_{F}|\overrightarrow{\mathrm{q}}| \gg \omega_{0}-\omega_{s}$ one can replace the sum over momenta in Eqs. (4.19), (4.20), and (5.11) according to

$$
\begin{aligned}
\sum_{\overrightarrow{\mathrm{p}} \in A_{m}} & \rightarrow \int_{\overrightarrow{\mathrm{p}} \in A_{m}} \frac{d \overrightarrow{\mathrm{p}}}{(2 \pi)^{3}} \rightarrow \frac{m^{2}}{(2 \pi)^{2} a^{2} q} \frac{A_{m}}{A} \int d \epsilon_{12}(\overrightarrow{\mathrm{p}}) d \epsilon_{12}(\overrightarrow{\mathrm{p}}+\overrightarrow{\mathrm{q}}) \\
& \rightarrow \frac{m^{2}}{(2 \pi)^{2} a^{2} q} \frac{A_{m}}{A} \int d E_{\alpha \beta}(\overrightarrow{\mathrm{p}}) d E_{\alpha \beta}(\overrightarrow{\mathrm{p}}+\overrightarrow{\mathrm{q}}) \frac{E_{\alpha \beta}(\overrightarrow{\mathrm{p}}) E_{\alpha \beta}(\overrightarrow{\mathrm{p}}+\overrightarrow{\mathrm{q}})}{\left[E_{\alpha \beta}^{2}(\overrightarrow{\mathrm{p}})-4 g^{2}\right]^{1 / 2}\left[E_{\alpha \beta}^{2}(\overrightarrow{\mathrm{p}}+\overrightarrow{\mathrm{q}})-4 g^{2}\right]^{1 / 2}} .
\end{aligned}
$$

With Eqs. (2.7) -(2.9), (4.14), (5.10), and (6.2)-(6.4) one obtains

$$
\begin{aligned}
& \frac{1}{\phi_{0}}\left(\frac{d^{2} \phi}{d \omega d \Omega}\right)_{N \alpha}=\frac{4 \omega_{s} \cos ^{2} \theta_{s}}{S \omega_{0} c^{4} \cos \theta_{0}} \frac{m^{2}}{(2 \pi)^{2} a^{2} q} \frac{A_{m}}{A} \sum_{\substack{\mu \nu \eta \xi \\
\mu^{\prime} \nu^{\prime} \eta^{\prime} \xi^{\prime}}} \int d E_{\alpha \beta}(\overrightarrow{\mathrm{p}}) d E_{\alpha \beta}(\overrightarrow{\mathrm{p}}+\overrightarrow{\mathrm{q}}) e_{s \mu} e_{s \nu} D_{\mu \mu^{\prime}}\left(\overrightarrow{\mathrm{k}}_{\|}, \omega_{s}\right) D_{\nu v^{\prime}}^{*}\left(\overrightarrow{\mathrm{k}}_{\|}, \omega_{s}\right) \\
& \times M_{\mu^{\prime} \eta^{\prime}}^{N}\left(\overrightarrow{\mathrm{p}}, \omega_{0}, \omega_{s} ; \alpha\right) M_{\nu^{\prime} \xi^{\prime}}^{N}\left(\overrightarrow{\mathrm{p}}, \omega_{0}, \omega_{s} ; \alpha\right) T_{\eta^{\prime} \eta} T_{\xi^{\prime} \xi}^{*} e_{0 \eta} e_{0 \xi} \\
& \times\left[f\left(g_{\alpha}\left(E_{\alpha \beta}(\overrightarrow{\mathrm{p}})\right)\right)-f\left(g_{\alpha}\left(E_{\alpha \beta}(\overrightarrow{\mathrm{p}}+\overrightarrow{\mathrm{q}})\right)\right)\right] \\
& \times \frac{E_{\alpha \beta}(\overrightarrow{\mathrm{p}}) E_{\alpha \beta}(\overrightarrow{\mathrm{p}}+\overrightarrow{\mathrm{q}})+4 g^{2}}{\left[E_{\alpha \beta}^{2}(\overrightarrow{\mathrm{p}})-4 g^{2}\right]^{1 / 2}\left[E_{\alpha \beta}^{2}(\overrightarrow{\mathrm{p}}+\overrightarrow{\mathrm{q}})-4 g^{2}\right]^{1 / 2}} \\
& \times \delta\left(\omega-F_{N \alpha}\left(E_{\alpha \beta}(\overrightarrow{\mathrm{p}}), E_{\alpha \beta}(\overrightarrow{\mathrm{p}}+\overrightarrow{\mathrm{q}})\right)\right), \\
& \frac{1}{\phi_{0}}\left(\frac{d^{2} \phi}{d \omega d \Omega}\right)_{N \beta}=\frac{4 \omega_{s} \cos ^{2} \theta_{s}}{S \omega_{0} c^{4} \cos \theta_{0}} \frac{m^{2}}{(2 \pi)^{2} a^{2} q} \frac{A_{m}}{A} \sum_{\substack{\mu \nu \eta \xi^{\prime} \\
\mu^{\prime} \nu^{\prime} \eta^{\prime} \xi^{\prime}}} \int d E_{\alpha \beta}(\overrightarrow{\mathrm{p}}) d E_{\alpha \beta}(\overrightarrow{\mathrm{p}}+\overrightarrow{\mathrm{q}}) e_{s \mu} e_{s \nu} D_{\mu \mu^{\prime}}\left(\overrightarrow{\mathrm{k}}_{\|,}, \omega_{s}\right) D_{\nu \nu^{\prime}}^{*}\left(\overrightarrow{\mathrm{k}}_{\|}, \omega_{s}\right) \\
& \times M_{\mu^{\prime} \eta^{\prime}}^{N}\left(\overrightarrow{\mathrm{p}}, \omega_{0}, \omega_{s} ; \beta\right) M_{\nu^{\prime} \xi^{\prime}}^{N}\left(\overrightarrow{\mathrm{p}}, \omega_{0}, \omega_{s} ; \beta\right) T_{\eta^{\prime} \eta} T_{\xi^{\prime} \xi}^{*} e_{0 \eta} e_{0 \xi} \\
& \times\left[f\left(g_{\beta}\left(E_{\alpha \beta}(\overrightarrow{\mathrm{p}}+\overrightarrow{\mathrm{q}})\right)\right)-f\left(g_{\beta}\left(E_{\alpha \beta}(\overrightarrow{\mathrm{p}})\right)\right)\right] \\
& \times \frac{E_{\alpha \beta}(\overrightarrow{\mathrm{p}}) E_{\alpha \beta}(\overrightarrow{\mathrm{p}}+\overrightarrow{\mathrm{q}})+4 g^{2}}{\left[E_{\alpha \beta}^{2}(\overrightarrow{\mathrm{p}})-4 g^{2}\right]^{1 / 2}\left[E_{\alpha \beta}^{2}(\overrightarrow{\mathrm{p}}+\overrightarrow{\mathrm{q}})-4 g^{2}\right]^{1 / 2}} \\
& \times \delta\left(\omega-F_{N \beta}\left(E_{\alpha \beta}(\overrightarrow{\mathrm{p}}), E_{\alpha \beta}(\overrightarrow{\mathrm{p}}+\overrightarrow{\mathrm{q}})\right)\right),
\end{aligned}
$$




$$
\begin{aligned}
& \frac{1}{\phi_{0}}\left(\left.\frac{d^{2} \phi}{d \omega d \Omega}\right|_{M}=\frac{4 \omega_{s} \cos ^{2} \theta_{s}}{S \omega_{0} c^{4} \cos \theta_{0}} \frac{m^{2}}{(2 \pi)^{2} a^{2} q} \frac{A_{m}}{A} \sum_{\substack{\mu \eta^{\prime} \xi \\
\mu^{\prime} \nu^{\prime} \xi^{\prime}}} \int d E_{\alpha \beta}(\overrightarrow{\mathrm{p}}) d E_{\alpha \beta}(\overrightarrow{\mathrm{p}}+\overrightarrow{\mathrm{q}}) e_{s \mu} e_{s \nu} D_{\mu \mu^{\prime}}\left(\overrightarrow{\mathrm{k}}_{\| l}, \omega_{s}\right) D_{\nu \nu \nu^{\prime}}^{*}\left(\overrightarrow{\mathrm{k}}_{\|,}, \omega_{s}\right)\right. \\
& \times M_{\mu^{\prime} \eta^{\prime}}^{M}\left(\overrightarrow{\mathrm{p}}, \omega_{0}, \omega_{s} ; \alpha, \beta\right) M_{\nu \xi^{\prime}}^{M^{*}}\left(\overrightarrow{\mathrm{p}}, \omega_{0}, \omega_{s} ; \alpha\right) T_{\eta^{\prime} \eta} T_{\xi^{\prime} \xi^{\prime}}^{*} e_{0 \eta^{\prime}} e_{0 \xi} \\
& \times\left[f\left(g_{\beta}\left(E_{\alpha \beta}(\overrightarrow{\mathrm{p}})\right)\right)-f\left(g_{\alpha}\left(E_{\alpha \beta}(\overrightarrow{\mathrm{p}}+\overrightarrow{\mathrm{q}})\right)\right)\right] \\
& \times \frac{E_{\alpha \beta}(\overrightarrow{\mathrm{p}}) E_{\alpha \beta}(\overrightarrow{\mathrm{p}}-\overrightarrow{\mathrm{q}})-4 g^{2}}{\left[E_{\alpha \beta}^{2}(\overrightarrow{\mathrm{p}})-4 g^{2}\right]^{1 / 2}\left[E_{\alpha \beta}^{2}(\overrightarrow{\mathrm{p}}+\overrightarrow{\mathrm{q}})-4 g^{2}\right]^{1 / 2}} \\
& \times \delta\left(\omega-F_{M}\left(E_{\alpha \beta}(\overrightarrow{\mathrm{p}}), E_{\alpha \beta}(\overrightarrow{\mathrm{p}}+\overrightarrow{\mathrm{q}})\right)\right)
\end{aligned}
$$

where

$$
\begin{aligned}
g_{\alpha}(x)= & \frac{1}{2} x+\frac{b}{2 a}\left(x^{2}-4 g^{2}\right)^{1 / 2} \\
g_{\beta}(x)= & \frac{1}{2} x-\frac{b}{2 a}\left(x^{2}-4 g^{2}\right)^{1 / 2} \\
F_{N \alpha}(x, y)= & \frac{1}{2} y-\frac{1}{2} x+\frac{b}{2 a}\left(y^{2}-4 g^{2}\right)^{1 / 2} \\
& -\frac{b}{2 a}\left(x^{2}-4 g^{2}\right)^{1 / 2}, \\
F_{N \beta}(x, y)= & \frac{1}{2} y-\frac{1}{2} x-\frac{b}{2 a}\left(y^{2}-4 g^{2}\right)^{1 / 2} \\
& +\frac{b}{2 a}\left(x^{2}-4 g^{2}\right)^{1 / 2}, \\
F_{M}(x, y)= & \frac{1}{2} y+\frac{1}{2} x+\frac{b}{2 a}\left(y^{2}-4 g^{2}\right)^{1 / 2} \\
& -\frac{b}{2 a}\left(x^{2}-4 g^{2}\right)^{1 / 2} .
\end{aligned}
$$$$
\frac{1}{\phi_{0}}\left(\frac{d^{2} \phi}{d \omega d \Omega}\right)_{N}=\frac{4 \omega_{s} \cos ^{2} \theta_{s}}{S \omega_{0} c^{4} \cos \theta_{0}} \frac{m^{2}}{(2 \pi)^{2} a^{2} q} \frac{A_{m}}{A}
$$$$
\times \sum_{\mu \nu \eta \xi} e_{s \mu} e_{s \nu} D_{\mu \mu^{\prime}}\left(\overrightarrow{\mathrm{k}}_{\|}, \omega_{s}\right) D_{\nu \nu^{\prime}}^{*}\left(\overrightarrow{\mathrm{k}}, \omega_{s}\right)\left(M_{\mu^{\prime} \eta^{\prime}}^{N \alpha} M_{\nu^{\prime} \xi}^{N \alpha^{*}}+M_{\mu^{\prime} \eta^{\prime}}^{N, \beta^{\prime}} M_{\nu^{\prime} \xi^{\prime}}^{N \xi^{*}}\right) T_{\eta^{\prime} \eta}^{*} T_{\xi^{\prime} \xi}^{*} e_{0 \eta} e_{0 \xi}
$$$$
\underset{\substack{\mu \nu \\ \mu \\ \mu} \nu^{\prime} \eta^{\prime} \xi^{\prime}}{ }
$$$$
\times \int_{g}^{\infty} d E[f(E)-f(\omega+E)] \frac{E(\omega+E)+g^{2}}{\left(E^{2}-g^{2}\right)^{1 / 2}\left[(\omega+E)^{2}-g^{2}\right]^{1 / 2}},
$$$$
\frac{1}{\phi_{0}}\left(\frac{d^{2} \phi}{d \omega d \Omega}\right)_{M}=\frac{4 \omega_{s} \cos ^{2} \theta_{s}}{S \omega_{0} c^{4} \cos \theta_{0}} \frac{m^{2}}{(2 \pi)^{2} a^{2} q} \frac{A_{m}}{A}
$$$$
\times \sum_{\substack{\mu \nu \eta \xi^{\prime} \nu^{\prime} \nu^{\prime} \\ \mu^{\prime} \nu^{\prime} \eta^{\prime} \xi^{\prime}}} e_{s \mu} e_{s \nu} D_{\mu \mu^{\prime}}\left(\overrightarrow{\mathrm{k}}_{\|}, \omega_{s}\right) D_{\nu \nu^{\prime}}^{*}\left(\overrightarrow{\mathrm{k}}_{\|}, \omega_{s}\right) M_{\mu \eta}^{M \beta \beta} M_{\nu}^{M \alpha \beta}{ }^{M} T_{\eta^{\prime} \eta} T_{\xi^{\prime} \xi}^{*} e_{0 \eta} e_{0 \xi}
$$$$
\times \int_{g}^{\omega-g} d E[1-f(E)-f(\omega-E)] \frac{E(\omega-E)-g^{2}}{\left(E^{2}-g^{2}\right)^{1 / 2}\left[(\omega-E)^{2}-g^{2}\right]^{1 / 2}} .
$$

In chromium $b<<a$ and one can write approximately

$$
\begin{aligned}
& g_{\alpha}\left(E_{\alpha \beta}(\overrightarrow{\mathrm{p}})\right)=g_{\beta}\left(E_{\alpha \beta}(\overrightarrow{\mathrm{p}})\right)=\frac{1}{2} E_{\alpha \beta}(\overrightarrow{\mathrm{p}}), \\
& F_{N \alpha}\left(E_{\alpha \beta}(\overrightarrow{\mathrm{p}}), E_{\alpha \beta}(\overrightarrow{\mathrm{p}}+\overrightarrow{\mathrm{q}})\right) \simeq \frac{1}{2}\left[E_{\alpha \beta}(\overrightarrow{\mathrm{p}}+\overrightarrow{\mathrm{q}})-E_{\alpha \beta}(\overrightarrow{\mathrm{p}})\right], \\
& F_{N \beta}\left(E_{\alpha \beta}(\overrightarrow{\mathrm{p}}), E_{\alpha \beta}(\overrightarrow{\mathrm{p}}+\overrightarrow{\mathrm{q}})\right) \simeq \frac{1}{2}\left[E_{\alpha \beta}(\overrightarrow{\mathrm{p}}+\overrightarrow{\mathrm{q}})-E_{\alpha \beta}(\overrightarrow{\mathrm{p}})\right], \\
& F_{M}\left(E_{\alpha \beta}(\overrightarrow{\mathrm{p}}), E_{\alpha \beta}(\overrightarrow{\mathrm{p}}+\overrightarrow{\mathrm{q}})\right) \simeq \frac{1}{2}\left[E_{\alpha \beta}(\overrightarrow{\mathrm{p}}+\overrightarrow{\mathrm{q}})+E_{\alpha \beta}(\overrightarrow{\mathrm{p}})\right] .
\end{aligned}
$$

If the band structure and the incident frequency $\omega_{0}$ are such that the matrix elements given by Eqs. (4.12), (4.13), and (5.9) could be assumed constant then, by using Eqs. (6.12)-(6.15), the integration over one energy variable in Eqs. (6.5)-(6.7) can be carried out easily, and one finds 
It is convenient to relate the scattering efficiency of the antiferromagnetic chromium to that in the normal metal.

For $M_{\mu \nu}^{n} \simeq M_{\mu \nu}^{N \alpha} \simeq M_{\mu \nu}^{N \beta} \simeq M_{\mu \nu}^{M \alpha \beta}=M$ the relative total scattering efficiency becomes

$$
\begin{aligned}
\frac{\tilde{\varphi}_{m}}{\tilde{\varphi}_{m}} & =\frac{\left[\left(d^{2} \phi / d \omega d \Omega\right)_{n}+\left(d^{2} \phi / d \omega d \Omega\right)_{N}+\left(d^{2} \phi / d \omega d \Omega\right)_{M}\right]_{T<T_{N}}}{\left[\left(d^{2} \phi / d \omega d \Omega\right)_{n}\right]_{T>T_{N}}} \\
& = \begin{cases}\frac{\left(I_{n}+I_{N}+I_{M}\right)_{T<T_{N}}}{\left(I_{n}\right)_{T>T_{N}}}, \text { for } \omega \geqslant 2 g, \\
\frac{\left(I_{n}+I_{N}\right)_{T<T_{N}}}{\left(I_{n}\right)_{T>T_{N}}}, \text { for } \omega<2 g,\end{cases}
\end{aligned}
$$

where

$$
\begin{aligned}
&\left(I_{n}\right)_{T>T_{N}}=\int_{0}^{\infty} d E[f(E)-f(\omega+E)] \\
&\left(I_{n}\right)_{T<T_{N}}=\frac{A_{m}}{A} \int_{0}^{\infty} d E[f(E)-f(\omega+E)] \\
&\left(I_{N}\right)_{T<T_{N}}=2 \frac{A_{m}}{A} \int_{g}^{\infty} d E[f(E)-f(\omega+E)] \\
& \times \frac{E(\omega+E)+g^{2}}{\left(E^{2}-g^{2}\right)^{1 / 2}\left[(\omega+E)^{2}-g^{2}\right]^{1 / 2}}
\end{aligned}
$$

$$
\begin{aligned}
\left(I_{M}\right)_{T<T_{N}}=\frac{A_{m}}{A} \int_{g}^{\omega-g} d E[1-f(E)-f(\omega-E)] \\
\times \frac{E(\omega-E)-g^{2}}{\left(E^{2}-g^{2}\right)^{1 / 2}\left[(\omega-E)^{2}-g^{2}\right]^{1 / 2}},
\end{aligned}
$$

and

$$
A=A_{n}+A_{m} .
$$

The integrals $I_{N}$ and $I_{M}$ can be solved numerically only.

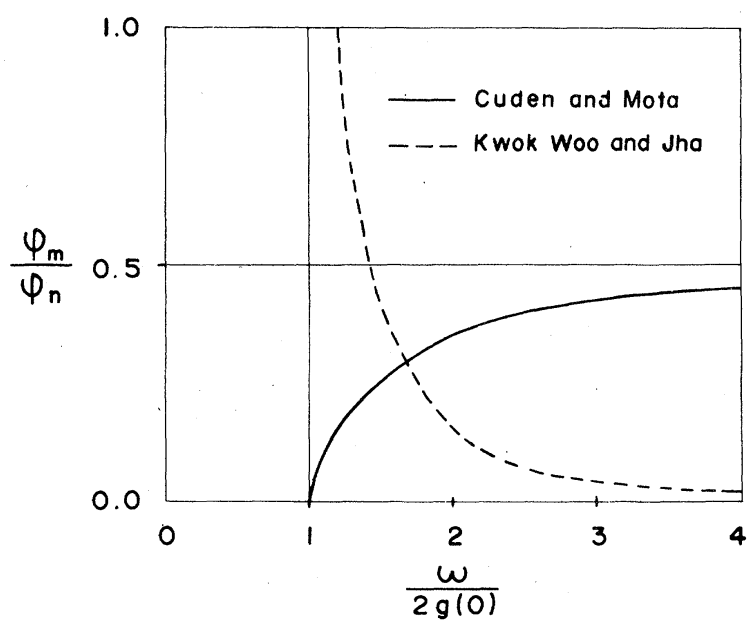

FIG. 4. Frequency dependence of relative scattering efficiency for antiferromagnetic chromium at $T=0^{\circ} \mathrm{K}$.
For $T=0^{\circ} \mathrm{K}$ Eq. (6:19) can be written in terms of complete eliptic integrals as

$$
\begin{aligned}
& \frac{\tilde{\varphi}_{m}}{\tilde{\varphi}_{n}} \\
& = \begin{cases}\frac{A_{m}}{A} \frac{2 g+\omega}{\omega} E\left(\frac{2 g-\omega}{2 g+\omega}\right)-\frac{A_{m}}{A} \frac{4 g}{\omega} K\left(\frac{2 g-\omega}{2 g+\omega}\right), \\
\quad \text { for } \omega \geqslant 2 g \\
0, \quad \text { for } \omega<2 g .\end{cases}
\end{aligned}
$$

The relative scattering efficiency at $T=0^{\circ} \mathrm{K}$ for the case of chromium $\left(2 g=3.5 k_{B} T_{N}, T_{N}=312^{\circ} \mathrm{K}\right.$, $\left.A_{m} / A=0.5\right)$ is shown in Fig. 4 .

\section{DISCUSSION}

The scattering efficiency obtained for the inelastic scattering of light in antiferromagnetic chromium shows no divergence at the threshold energy equal to twice the antiferromagnetic energy gap and exhibits completely different frequency dependence to that found in Ref. 21. This is the consequence of properly treating the coherence effects in our calculation.

In conclusion, let us mention the approximations made in our calculation and the mechanisms which could affect the frequency and temperature dependence of the scattering efficiency.

The effective interaction and the energy gap are assumed constant over the magnetic part of the Fermi surface. Further, we assumed, that in chromium, there is a good matching of electron-hole pockets.

Interactions between the quasiparticles created in the scattering are ignored.

Smearing of the Fermi surface and the depairing of bound-electron-hole pairs by random fluctuating electric fields, caused by the electron-phonon interaction, is neglected.

In the present calculation of the scattering efficiency the model Hamiltonian that leads to the second- 
order phase transition is used. As is well known, chromium exhibits first-order phase transition and therefore one cannot rely on the results of our calculation in the temperature region close to the critical temperature, $T_{N}$.

The calculation of the scattering efficiency based on the more realistic band model of chromium is in progress.

Finally, let us point out that by using the Ramanscattering technique one can estimate $A_{m} / A$ and ob- tain a better value for the order parameter than by other indirect methods.

\section{ACKNOWLEDGMENT}

One of the authors (R.M.) would like to kindly thank Coordenação de Aperfeiçoamento de Pessoal de Nível Superior (CAPES) for a graduate fellowship. This work was partially supported by the Financiadora de Estudos e Projetos (FINEP) of Brasil.
"Part of the thesis required for the partial fulfilment of M.Sc degree at the Universidade Federal da Bahia, Departamento de Física do Estado Sólido.

${ }^{1}$ A. Smekal, Naturwiss. 11,873 (1923).

${ }^{2}$ M. S. Khaikin and V. P. Bykov, Zh. Eksp. Teor. Fiz. 30, 191 (1956).

${ }^{3}$ A. Mooradian, Phys. Rev. Lett. 20, 1102 (1968).

${ }^{4}$ P. A. Wolf, Phys. Rev. 171, 436 (1968).

${ }^{5}$ P. M. Platzman, Phys. Rev. 139, A379 (1965).

${ }^{6}$ D. W. Feldman, J. H. Parker, and M. Ashkin, Phys. Rev. Lett. 21, 607 (1968).

${ }^{7}$ D. L. Mills, A. A. Maradudin, and E. Burstein, Phys. Rev. Lett. 21, 1178 (1968); and Ann. Phys. (N.Y.) $\underline{56}, 504$ (1970).

${ }^{8}$ A. Mooradian, Phys. Rev. Lett. 22, 185 (1969).

9J. F. Scott, R. F. Leheney, J. P. Remeika, and A. R. Sweedler, Phys. Rev. B 2, 3883 (1970).

${ }^{10}$ D. R. Tilley, J. Phys. F $\underline{3}, 1417$ (1973).

${ }^{11}$ L. M. Fraas, P. F. Williams, and S. P. S. Porto, Solid State Commun. 8,2113 (1970).

${ }^{12}$ A. A. Abrikosov and L. A. Falkovskii, Zh. Eksp. Teor. Fiz. 40, 262 (1961) [Sov. Phys. JETP 13, 179 (1961)].
${ }^{13}$ A. A. Maradudin, Mater. Res. Bull. 4, 563 (1969).

${ }^{14}$ D. R. Tilley, Z. Phys. 254, 71 (1972).

${ }^{15}$ F. G. Dos Reis and R. Luzzi, Rev. Brasileira Fis. $\underline{3}$, 531 (1973).

${ }^{16}$ C. B. Cuden, Phys. Rev. B $\underline{18}, 3156$ (1978).

${ }^{17}$ C. B. Cuden, Phys. Rev. B $\underline{13}, 1993$ (1976).

${ }^{18}$ C. B. Cuden, Phys. Rev. (to be published).

${ }^{19}$ S. S. Jha, Phys. Rev. 182, 813 (1969).

${ }^{20}$ D. C. Hamilton and A. L. McWhorter, Proceedings of the International Conference on Light Scattering Spectra of Solids, New York, 1968, edited by George B. Wright (SpringerVerlag, New York, 1969).

${ }^{21}$ P. C. Kwok, James W. F. Woo, and Sudhanshu S. Jha, Phys. Rev. 182, 671 (1969).

${ }^{22}$ W. M. Lomer, Proc. Phys. Soc. London 80, 489 (1962).

${ }^{23}$ W. C. Koehler, R. M. Moon, A. L. Trego, and A. R. Mackintosh, Phys. Rev. 151, 405 (1966).

${ }^{24}$ L. F. Mattheiss, Phys. Rev. 139, A1893 (1965).

${ }^{25}$ T. L. Loucks, Phys. Rev. 139, A1181 (1965).

${ }^{26}$ A. W. Overhauser, Phys. Rev. 128, 1437 (1962).

${ }^{27}$ P. A. Fedders and P. C. Martin, Phys. Rev. 143, 245 (1965).

${ }^{28}$ A. Kawabata, J. Phys. Soc. Jpn. 29, 890 (1970). 